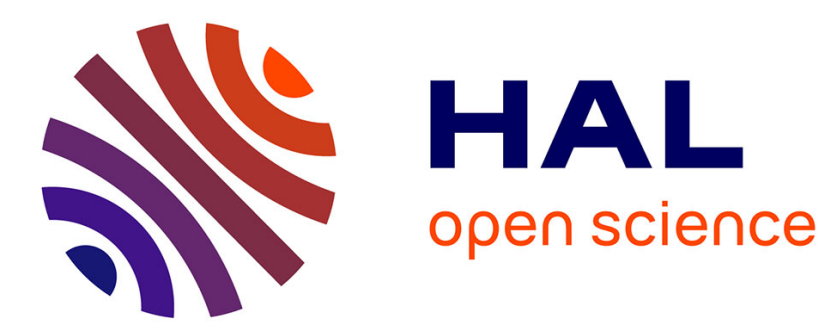

\title{
Recognizability for automata
}

Didier Caucal, Chloé Rispal

\section{- To cite this version:}

Didier Caucal, Chloé Rispal. Recognizability for automata. DLT 2018, Sep 2018, Tokyo, Japan. pp.206-218, 10.1007/978-3-319-98654-8_17 . hal-01824290

\section{HAL Id: hal-01824290 \\ https://hal.science/hal-01824290}

Submitted on 27 Jun 2018

HAL is a multi-disciplinary open access archive for the deposit and dissemination of scientific research documents, whether they are published or not. The documents may come from teaching and research institutions in France or abroad, or from public or private research centers.
L'archive ouverte pluridisciplinaire HAL, est destinée au dépôt et à la diffusion de documents scientifiques de niveau recherche, publiés ou non, émanant des établissements d'enseignement et de recherche français ou étrangers, des laboratoires publics ou privés. 


\title{
Recognizability for automata
}

\author{
Didier Caucal and Chloé RIspal \\ LIGM-CNRS University Paris-Est \\ $\{$ caucal,rispal\}@u-pem.fr
}

\begin{abstract}
We present a new approach to define boolean algebras of various language families : given a family $\mathcal{F}$ of infinite automata, an automaton $H$ recognizes the set of languages accepted by all automata of $\mathcal{F}$ that can be mapped by morphism into $H$. Considering appropriate automata families, we get boolean algebras of context-free languages, indexed languages, Petri net languages, higher order indexed languages and context-sensitive languages.
\end{abstract}

\section{Introduction}

The family of regular languages is closed under many operations. Those closure properties give an easy way to work with this family and specially the closure under boolean operations. Some of these boolean closure properties are not satisfied at the next level of the Chomsky hierarchy: the family of context-free languages is not closed under complementation and intersection, and the subfamily of deterministic context-free languages is not closed under union and intersection. This boolean closure properties are satisfied neither by the families of Petri net languages and higher-order indexed languages [11].

In this paper, we present a new approach to extract boolean algebras of all those language families. A standard way to get boolean algebras is by recognizability by inverse morphism as defined by Eilenberg $[5,6]$ for any monoid. This notion of recognizability has been extended to terms, finite graphs, pictures, traces, words indexed by linear orderings (see [17] among others). In all of these extensions, recognizability is associated to appropriate finite automata. In this paper, we adapt recognizability by inverse morphism to infinite automata.

An automaton is a set of labelled edges with some initial and final vertices. A morphism $f$ from an automaton $G$ into an automaton $H$ is a mapping from the vertices of $G$ into the vertices of $H$ such that for any edge $s \stackrel{a}{\longrightarrow} t$ of $G$, $f(s) \stackrel{a}{\longrightarrow} f(t)$ is an edge of $H$ and for $s$ initial/final in $G, f(s)$ is initial/final in $H$. We define the recognizability by an automaton $H$ according to an automaton family $\mathcal{F}$ as the set $\operatorname{Rec}_{\mathcal{F}}(H)$ of languages accepted by the automata of $\mathcal{F}$ that can be mapped by morphism into $H$.

A way to obtain boolean algebras of context-free languages is by synchronization of pushdown automata [13,4]. This approach corresponds to the recognizability with morphisms preserving the stack height. We show that this notion can not be generalized to get boolean algebra for stack of stack automata. 
We consider the family $\mathcal{F}(\mathcal{R})$ of automata such that each labelled transition $\stackrel{a}{\longrightarrow}$ is a binary relation of a set $\mathcal{R}$. We introduce the structural recognizability according to $\mathcal{F}(\mathcal{R})$ by restricting the morphisms to relations of $\mathcal{R}$. To get the closure under complementation, we add a natural condition of determinism on the morphisms. Structural recognizability defines boolean algebras for pushdown automata and also for stack of stack automata. More generally, we consider the hierarchy of multistack of stack automata. We prove that for any automaton family of this hierarchy, structural recognizability and deterministic structural recognizability coincide and define a boolean algebra for any unambiguous automaton. We also get boolean algebras by deterministic structural recognizability of synchronized automata.

\section{Recognizability}

In this section, we give notations and we define recognizability by inverse morphism for infinite automata.

\subsection{Automata}

An automaton is a labelled oriented simple graph with initial and final vertices. More precisely, an automaton $G$ is defined by $G \subseteq \mathrm{V}_{G} \times \mathrm{T}_{G} \times \mathrm{V}_{G} \cup\{\iota, o\} \times \mathrm{V}_{G}$ where $\mathrm{V}_{G}$ is a finite or countable set of vertices, $\mathrm{T}_{G}$ is a finite set of labels, $\iota$ marks the initial vertices, and $o$ marks the final vertices. Any triple $(s, a, t) \in G$ is an edge labelled by a from source $s$ to goal $t$; it is also denoted by $s \stackrel{a}{\longrightarrow} t$ i.e. $\stackrel{a}{\longrightarrow}_{G}=\left\{(s, t) \mid s \stackrel{a}{\longrightarrow}_{G} t\right\}$ is the a-transition of $G$. Any couple $(c, s) \in G$ is a vertex coloured by $c \in\{\iota, o\}$; it is denoted by $c s \in G$ and $\stackrel{c}{\longrightarrow} G=$ $\{(s, s) \mid c s \in G\}$ is the $c$-transition of $G$.

Taking symbols $\mid, \kappa$, and a triple $\vec{T}=\left(T_{-1}, T_{0}, T_{1}\right)$ of disjoint alphabets, we define the input-driven automaton:

$$
\begin{aligned}
& \operatorname{Inp}_{1,1}(\vec{T})=\left\{\left.\left.\quad\right|^{n} \kappa \stackrel{a}{\longrightarrow}\right|^{n+1} \kappa \mid n \geq 0 \wedge a \in T_{1}\right\} \\
& \cup\left\{\left.\left.\right|^{n+1} \kappa \stackrel{b}{\longrightarrow}\right|^{n} \kappa \quad \mid n \geq 0 \wedge b \in T_{-1}\right\} \\
& \cup\left\{\left.\left.\right|^{n} \kappa \stackrel{c}{\longrightarrow}\right|^{n} \kappa \quad \mid n \geq 0 \wedge c \in T_{0}\right\} \cup\{\iota \kappa\} \cup\left\{\left.o\right|^{n} \kappa \mid n \geq 0\right\} \text {. }
\end{aligned}
$$

The automaton $\operatorname{Inp}_{1,1}(\{b\},\{c\},\{a\})$ is represented below.

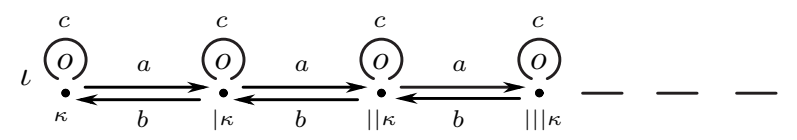

Recall that a path $s_{0} \stackrel{a_{1}}{\longrightarrow} \ldots \stackrel{a_{n}}{\longrightarrow} s_{n}$ is a sequence of consecutive transitions and we write $s_{0} \underset{G}{\stackrel{a_{1} \ldots a_{n}}{\rightarrow}} s_{n}$; such a path is accepting for $s_{0}$ initial and $s_{n}$ final. The language accepted by an automaton $G$ is the set $\mathrm{L}(G)$ of labels of the accepting paths: 


$$
\mathrm{L}(G)=\left\{u \in \mathrm{T}_{G}^{*} \mid \exists s, t(s \underset{G}{\stackrel{u}{\longrightarrow}} t \wedge \iota s, o t \in G)\right\} .
$$

The previous automaton $\operatorname{Inp}_{1,1}(\{b\},\{c\},\{a\})$ accepts the language

$$
\mathrm{L}\left(\operatorname{Inp}_{1,1}(\{b\},\{c\},\{a\})\right)=\left\{\left.u \in\{a, b, c\}^{*}|\forall v \leq u,| v\right|_{a} \geq|v|_{b}\right\}
$$

of prefixes of well-parenthesed words ( $a$ the open parenthesis and $b$ the close one). The notation $v \leq u$ means that $u$ is a prefixe of $v$. An automaton is deterministic if it has a unique initial vertex and if for any vertex $s$ and any label $a$, there exists at most one transition starting from $s$ and labelled by $a$. More generally, an automaton is unambiguous, if any two accepting paths have distinct labels. The previous automata $\operatorname{Inp}_{1,1}(\vec{T})$ are deterministic.

A morphism $f$ from an automaton $G$ into an automaton $H$ is a mapping $f: \mathrm{V}_{G} \longrightarrow \mathrm{V}_{H}$ such that for any $s, t \in \mathrm{V}_{G}, a \in \mathrm{T}_{G}$ and $c \in\{\iota, o\}$,

$$
s \underset{G}{\stackrel{a}{\longrightarrow}} t \Longrightarrow f(s) \underset{H}{\stackrel{a}{\longrightarrow}} f(t) \text { and } \quad c s \in G \Longrightarrow c f(s) \in H
$$

we write $G \stackrel{f}{\longrightarrow} H$ and say that $G$ is $f$-reducible into $H$.

\subsection{Recognizability}

Recall that a language family $\mathcal{L}$ is a boolean algebra with respect to a language $L \in \mathcal{L}$ if

$$
P \subseteq L \text { and } L-P, P \cap Q \in \mathcal{L} \text { for any } P, Q \in \mathcal{L} .
$$

We define recognizability for families $\mathcal{F}$ of automata to get boolean sub-algebras of

$$
\mathcal{L}(\mathcal{F})=\{\mathrm{L}(G) \mid G \in \mathcal{F}\} .
$$

For any fixed monoid $M$, Eilenberg has defined the following family $\operatorname{rec}(M)$ of recognizable subsets of $M$ by inverse morphism:

$$
\left\{f^{-1}(P) \mid \exists N \text { finite monoid }(f: M \rightarrow N \text { morphism } \wedge P \subseteq N)\right\} .
$$

Contrary to Eilenberg, we define recognizability by fixing the image of the morphism while its input domain takes values in a family of automata. Precisely, let $\mathcal{F}$ be a family of automata and let $H$ be an automaton not necessarily in $\mathcal{F}$. We consider the set of languages accepted by all possible automata $G$ of $\mathcal{F}$ reducible into $H$ :

$$
\operatorname{Rec}_{\mathcal{F}}(H)=\{\mathrm{L}(G) \mid G \in \mathcal{F} \wedge G \longrightarrow H\} .
$$

Denote by $\operatorname{Loop}_{T}$ the reduced automaton with one state and the loops labelled by each letter of the alphabet $T$ :

$$
\operatorname{Loop}_{T}=\{\kappa \stackrel{a}{\longrightarrow} \kappa \mid a \in T\} \cup\{\iota \kappa, o \kappa\} .
$$

Any automaton labelled in $T$ can be reduced to $\operatorname{Loop}_{T}$ hence

$$
\operatorname{Rec}_{\mathcal{F}}\left(\operatorname{Loop}_{T}\right)=\mathcal{L}(\mathcal{F}) .
$$


Proposition 2.1 For the family $\mathcal{F}_{0}$ of finite automata and for any $H \in \mathcal{F}_{0}$,

$$
\operatorname{Rec}_{\mathcal{F}_{0}}(H)=\{L \subseteq \mathrm{L}(H) \mid L \text { regular }\}
$$

is a boolean algebra with respect to $\mathrm{L}(H)$.

In particular $\operatorname{Rec}_{\mathcal{F}_{0}}\left(\operatorname{Loop}_{T}\right)$ is the set $\operatorname{Reg}\left(T^{*}\right)$ of regular languages over $T$ which is a boolean algebra. This is not true in general for any family $\mathcal{F}$ of automata. So conditions have to be given on the morphisms to get boolean algebras.

\section{Length recognizability}

Boolean algebras of context-free languages can already be obtained by synchronization of pushdown automata $[13,4]$. This notion corresponds to recognizability by morphisms preserving vertex length. In the case of pushdown automata, length recognizability defines a boolean algebra of context-free languages for any unambiguous pushdown automaton.

We show that this approach is not suitable any more when considering stack of stack automata: the set of languages accepted by stack of stack automata mapped by a length-preserving morphism into a deterministic stack of stack automaton is not in general closed under complementation.

\subsection{Families of automata on words}

We define an automaton family by the type of relations of its labelled transitions. Given a family $\mathcal{R}$ of binary relations, we define the family $\mathcal{F}(\mathcal{R})$ of automata $G$ whose labelled transitions are relations of $\mathcal{R}$ :

$$
\underset{G}{\stackrel{a}{\longrightarrow}} \in \mathcal{R} \text { for any } a \in \mathrm{T}_{G} \cup\{\iota, o\} .
$$

From now on, $N$ is a countable set of symbols. We denote by

$$
\mathcal{R}_{0}=\{R \subseteq N \times N \mid R \text { finite }\}
$$

the family of finite binary relations on $N$. So $\mathcal{F}\left(\mathcal{R}_{0}\right)$ is the set of finite automata having vertices in $N$. For instance, for the family $\mathcal{R}_{1}$ of relations of the form

$$
\left\{\left(\left|{ }^{n} \kappa,\right|^{n+1} \kappa\right) \mid n \in P\right\} \quad \text { or }\left\{\left(\left|{ }^{n+1} \kappa,\right|^{n} \kappa\right) \mid n \in P\right\} \text { or }\left\{\left({ }^{n} \kappa,\left.\right|^{n} \kappa\right) \mid n \in P\right\}
$$

where $P$ is a regular subset of $\mathbb{N}$, the automata $\operatorname{Inp}_{1,1}(\vec{T})$ and $\operatorname{Loop}_{T}$ are in $\mathcal{F}\left(\mathcal{R}_{1}\right)$.

\subsection{Length recognizability for pushdown automata}

A morphism $f$ from an automaton $G$ is length-preserving if for any vertex $s \in V_{G}$, $|f(s)|=|s|$. For any family $\mathcal{R}$ of binary relations on $N^{*}$ and for any automaton $H$ of vertex set $\mathrm{V}_{H} \subseteq N^{*}$, the length recognizability is

$$
\begin{aligned}
& \ell \operatorname{Rec}_{\mathcal{F}(\mathcal{R})}(H) \\
= & \{\mathrm{L}(G) \mid G \in \mathcal{F}(\mathcal{R}) \wedge \exists f(G \stackrel{f}{\longrightarrow} H \wedge f \text { length-preserving })\}
\end{aligned}
$$


the restriction of recognizability to length-preserving morphisms.

In particular the family of input-driven languages [12] for any triple $\vec{T}$ of disjoint alphabets and for any family $\mathcal{R}$ of binary relations on $N^{*}$ is

$$
\operatorname{Idl}_{\mathcal{R}}(\vec{T})=\ell \operatorname{Rec}_{\mathcal{F}(\mathcal{R})}\left(\operatorname{Inp}_{1,1}(\vec{T})\right) .
$$

So, for a triple $\vec{T}=\left(T_{-1}, T_{0}, T_{1}\right)$, a language is input-driven if it is accepted by an automaton such that for $u \stackrel{a}{\longrightarrow} v$, we have $(|v|=|u|+i)$ for $a \in T_{i}$ and $(|u|=0$ for $a=\iota$ ).

Length recognizability has been already considered for the family of pushdown automata. Let us recall the suffix relations which define the same automaton family as pushdown automata restricted to a regular vertex set [3]. The left concatenation of a language $W \subseteq N^{*}$ with a binary relation $R \subseteq N^{*} \times N^{*}$ is the relation

$$
W \cdot R=\{(w u, w v) \mid w \in W \wedge(u, v) \in R\} .
$$

For instance $N^{*} \cdot R$ is the suffix rewriting of $R$. For words $u, v$, we denote by

$$
W(u, v)=W \cdot\{(u, v)\}=\{(w u, w v) \mid w \in W\}
$$

and is called an elementary suffix relation when $W$ is a regular language. A suffix relation is a finite union of elementary suffix relations:

$$
W_{1}\left(u_{1}, v_{1}\right) \cup \ldots \cup W_{n}\left(u_{n}, v_{n}\right) \text { with } n \geq 0 \text { and } W_{1}, \ldots, W_{n} \text { regular. }
$$

The family of suffix relations is denoted by $\mathcal{S}$ tack.

For instance the automaton $H_{1}$ defined by the suffix relations:

$$
\begin{array}{rlrl}
\stackrel{a}{\longrightarrow} & =\left.\right|^{*}(\varepsilon, \mid) & ; & \stackrel{b}{\longrightarrow}=\left.\left.\right|^{*}(\mid, \varepsilon) \cup\right|^{*} \dagger^{*}(\varepsilon, \dagger) \\
\stackrel{c}{\longrightarrow}=\left.\right|^{*} \dagger^{*}(\dagger, \varepsilon) & ; \quad \stackrel{o}{\longrightarrow}=\stackrel{\iota}{\longrightarrow}=(\varepsilon, \varepsilon)
\end{array}
$$

belongs to $\mathcal{F}(\mathcal{S}$ tack $)$ and is represented as follows:

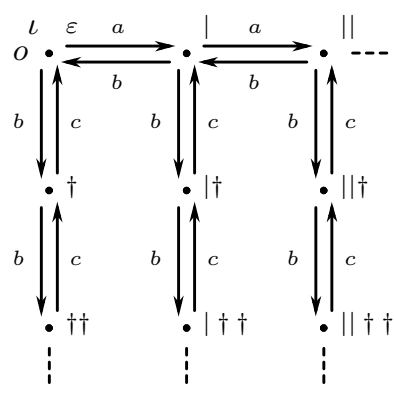

Length recognizability for pushdown automata corresponds to synchronization and defines a boolean algebra of context-free languages for any deterministic pushdown automaton [13] and for any unambiguous pushdown automaton [4].

Theorem 1. For any unambiguous automaton $H \in \mathcal{S}$ tack,

$\ell \operatorname{Rec}_{\mathcal{F}(\mathcal{S} \text { tack })}(H)$ is a boolean algebra with respect to $\mathrm{L}(H)$.

In particular for any partition $\vec{T}$, the family $\operatorname{Idl}_{\mathcal{S} \text { tack }}(\vec{T})$ of input-driven pushdown languages is a boolean algebra with respect to $\mathrm{L}\left(\operatorname{Inp}_{1,1}(\vec{T})\right)[12]$. 


\subsection{Length recognizability for stack of stack automata}

So length recognizability defines boolean algebras for pushdown automata but this approach is not suitable anymore when considering stack of stack automata. We present the suffix relations of level 2, defining the same automaton family as stack of stack automata. A vertex of a stack of stack automaton is a word of words that is a tuple of words:

$$
\vec{x}=\left(x_{0}, \ldots, x_{k}\right) \in\left(N^{*}\right)^{k} \text { with } k \geq 0 ;
$$

the word $x_{k}$ corresponds to the topmost stack of level 1 .

The following elementary operations are defined on words of words:

for any $a \in N$ and $x_{0}, \ldots, x_{k} \in N^{*}$,

We denote by

$$
\begin{aligned}
& \left(x_{0}, \ldots, x_{k}\right) \stackrel{a}{\longrightarrow}\left(x_{0}, \ldots, x_{k} a\right) \quad \stackrel{\bar{a}}{\longrightarrow}\left(x_{0}, \ldots, x_{k}\right) \\
& \left(x_{0}, \ldots, x_{k}\right) \stackrel{\#}{\longrightarrow}\left(x_{0}, \ldots, x_{k}, x_{k}\right) \stackrel{\#}{\longrightarrow}\left(x_{0}, \ldots, x_{k}\right) \\
& (\varepsilon) \stackrel{!}{\longrightarrow}(\varepsilon) \quad\left(x_{0}, \ldots, x_{k-1}, \varepsilon\right) \stackrel{?}{\longrightarrow}\left(x_{0}, \ldots, x_{k-1}, \varepsilon\right)
\end{aligned}
$$

$$
O p=N \cup \bar{N} \cup\{\#, \#, !, ?\}
$$

the set of letters representing those operations.

Elementary relations on words of operations are of the form:

$$
W(u, v)=\{(w u, w v) \mid w \in W\} \subseteq O p^{*} \times O p^{*}
$$

where $W$ is a regular set over $O p$.

Elementary suffix relations of level 2 (on words of words) are defined by interpretation:

$$
\llbracket W(u, v) \rrbracket=\{(\vec{x}, \vec{y}) \mid \exists w \in W, \exists \vec{z},(\varepsilon) \stackrel{w}{\longrightarrow} \vec{z} \wedge \vec{z} \stackrel{u}{\longrightarrow} \vec{x} \wedge \vec{z} \stackrel{v}{\longrightarrow} \vec{y}\}
$$

where the context $W$ is a regular subset of $O p^{*}, u$ and $v$ are words over $O p$, and $\vec{x}, \vec{y}, \vec{z}$ are words of words over $N$. In particular

$$
\stackrel{w}{\longrightarrow}=\llbracket O p^{*}(\varepsilon, w) \rrbracket \text { for any } w \in O p^{*} .
$$

A suffix relation of level 2 is a finite union of elementary suffix relations of level 2 and the set of suffix relations of level 2 is denoted by $\mathcal{S}$ tack $_{2}$. These suffix relations can be normalized [2]. The languages accepted by automata of $\mathcal{F}\left(\mathcal{S}\right.$ tack $\left._{2}\right)$ are indexed languages [1] but the indexed language

$$
\left\{u u \mid u \in\{a, b\}^{*}\right\} \notin \mathcal{L}\left(\mathcal{F}\left(\mathcal{S} \text { tack }_{2}\right)\right) \text {. }
$$

For instance, the following automata of $\mathcal{F}\left(\mathcal{S t a c k}_{2}\right)$ are a natural extention of the automata $\operatorname{Inp}_{1,1}$. For any quintuple $\vec{T}=\left(T_{-1}, T_{0}, T_{1}, T_{-1}^{\prime}, T_{1}^{\prime}\right)$ of disjoint alphabets, the automaton $\operatorname{Inp}_{1,2}(\vec{T})$ is defined for any $a \in T_{1}, b \in T_{-1}, c \in T_{0}$, $A \in T_{1}^{\prime}$ and $B \in T_{-1}^{\prime}$ by

$$
\begin{aligned}
& \stackrel{a}{\longrightarrow}=\llbracket o p^{*}(\kappa, \mid \kappa) \rrbracket=\left\{\left(\left.\right|^{n_{0}}, \ldots,\left.\right|^{n_{k}}\right) \kappa \rightarrow\left(\left.\right|^{n_{0}}, \ldots,\left.\right|^{n_{k}+1}\right) \kappa \mid k, n_{0}, \ldots, n_{k} \geq 0\right\} \\
& \stackrel{b}{\longrightarrow}=\llbracket o p^{*}(\mid \kappa, \kappa) \rrbracket=\left\{\left(\left.\right|^{n_{0}}, \ldots,\left.\right|^{n_{k}+1}\right) \kappa \rightarrow\left(\left.\right|^{n_{0}}, \ldots,\left.\right|^{n_{k}}\right) \kappa \mid k, n_{0}, \ldots, n_{k} \geq 0\right\} \\
& \stackrel{c}{\longrightarrow}=\llbracket o p^{*}(\kappa, \kappa) \rrbracket=\left\{\left(\left.\right|^{n_{0}}, \ldots,\left.\right|^{n_{k}}\right) \kappa \rightarrow\left(\left.\right|^{n_{0}}, \ldots,\left.\right|^{n_{k}}\right) \kappa \mid k, n_{0}, \ldots, n_{k} \geq 0\right\} \\
& \stackrel{A}{\longrightarrow}=\llbracket o p^{*}(\kappa, \# \kappa) \rrbracket=\left\{\left(\left.\right|^{n_{0}}, \ldots,\left.\right|^{n_{k}}\right) \kappa \rightarrow\left(\left.\right|^{n_{0}}, \ldots,\left.\right|^{n_{k}},\left.\right|^{n_{k}}\right) \kappa \mid k, n_{0}, \ldots, n_{k} \geq 0\right\}
\end{aligned}
$$




$$
\begin{aligned}
& \left.\stackrel{B}{\longrightarrow}=\llbracket o p^{*}(\# \kappa, \kappa) \rrbracket=\left\{\left(\left.\right|^{n_{0}}, \ldots,||^{n_{k}},\left.\right|^{n_{k}}\right) \kappa \rightarrow\left(||^{n_{0}}, \ldots, \mid{ }^{n_{k}}\right) \kappa\right) \mid k, n_{0}, \ldots, n_{k} \geq 0\right\} \\
& \stackrel{\iota}{\longrightarrow}=\llbracket(\kappa, \kappa) \rrbracket \quad=\{(\kappa, \kappa)\} \\
& \stackrel{o}{\longrightarrow}=\llbracket o p^{*}(\kappa, \kappa) \rrbracket=\left\{\left(\left.\right|^{n_{0}}, \ldots,\left.\right|^{n_{k}}\right) \kappa \rightarrow\left(\left.\right|^{n_{0}}, \ldots,\left.\right|^{n_{k}}\right) \kappa \mid k, n_{0}, \ldots, n_{k} \geq 0\right\}
\end{aligned}
$$

where $o p=\{\mid, \overline{\mid}, \#, ?\}$ and $\left(\left.\right|^{n_{0}}, \ldots,\left.\right|^{n_{k}}\right) \kappa=\left(\left|{ }^{n_{0}}, \ldots,\right|^{n_{k}} \kappa\right)$.

For instance, $\operatorname{Inp}_{1,2}(\{b\},\{c\},\{a\},\{B\},\{A\})$ is represented below

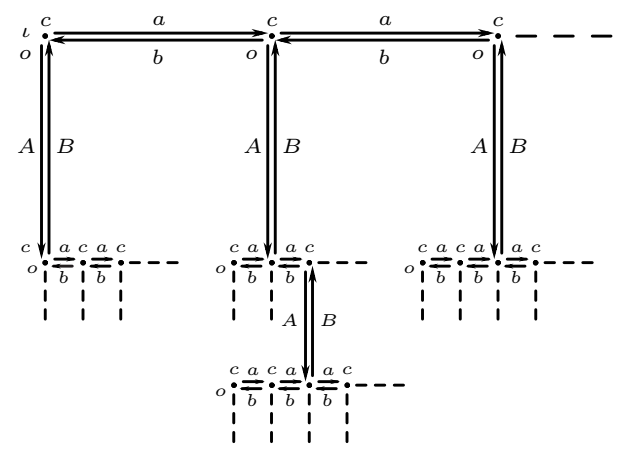

and corresponds to the Muchnik's operation [15] of $\operatorname{Inp}_{1,1}(\{b\},\{c\},\{a\})$.

Length recognizability is naturally extended to automata whose vertices are words of words. Precisely a function $f:\left(N^{*}\right)^{*} \longrightarrow\left(N^{*}\right)^{*}$ on words of words is length-preserving if the image by $f$ preserves the number of components and the length of each component: for each $f\left(x_{1}, \ldots, x_{m}\right)=\left(y_{1}, \ldots, y_{n}\right)$,

$$
m=n \wedge\left|x_{1}\right|=\left|y_{1}\right| \wedge \ldots \wedge\left|x_{m}\right|=\left|y_{m}\right| .
$$

Length recognizability does not define in general boolean algebras for stack of stack automata. For instance, a natural extension of input-driven languages is not closed under complementation.

Proposition 3.1 For any partition $\vec{T}$ of non empty alphabets, $\ell \operatorname{Rec}_{\mathcal{F}\left(\mathcal{S t a c k}_{2}\right)}\left(\operatorname{Inp}_{1,2}(\vec{T})\right)$ is not closed under complementation.

As a counter example, the language

$$
\begin{aligned}
\left\{a^{n} c b^{n_{1}} a^{n_{1}} d \ldots c b^{n_{k}} a^{n_{k}} d \mid\right. & n, k>0 \wedge 0<n_{1}, \ldots, n_{k} \leq n \\
& \left.\wedge \exists 1 \leq i<j \leq k, n_{i}=n_{j}\right\}
\end{aligned}
$$

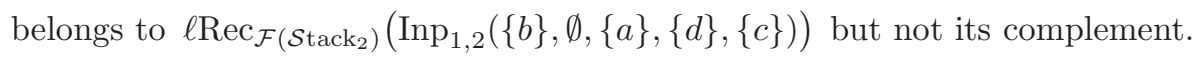

\section{Structural recognizability}

Since length recognizability does not define boolean algebras beyond context-free languages, we introduce a new recognizability. For any automaton family $\mathcal{F}(\mathcal{R})$, structural recognizability requires the morphism to be a relation of $\mathcal{R}$.

Like for finite automata, we need determinism to get boolean algebras by structural recognizability but we do not determinize automata : we introduce a condition of determinism on the morphisms. When the morphisms are deterministic 
and belongs to $\mathcal{R}$, recognizability is deterministic structural.

In this section, we apply structural recognizability to the whole hierarchy of multi-stack of stack automata. We prove that for the family of automata defined by $m$ stacks of level $n$, structural recognizability and deterministic structural recognizability coincide and define a boolean algebra for any unambiguous automaton.

For any automaton family $\mathcal{F}(\mathcal{R})$, the recognizability is structural when the morphism is a relation of $\mathcal{R}$.

Definition 1. We define by structural recognizability from an automaton $H$ according to an automaton family $\mathcal{F}(\mathcal{R})$ the set

$$
\operatorname{sRec}_{\mathcal{F}(\mathcal{R})}(H)=\{\mathrm{L}(G) \mid G \in \mathcal{F}(\mathcal{R}) \wedge \exists f \in \mathcal{R}(G \stackrel{f}{\longrightarrow} H)\} .
$$

To illustrate this definition, we introduce the family of one counter relations defined by pushdown automata using a unique stack symbol $\mid \in N$.

An elementary one counter relation is of the form

$$
W(u, v)=\{(w u, w v) \mid w \in W\} \text { for } W \in \operatorname{Reg}\left(\left.\right|^{*}\right) \text { and } u,\left.v \in\right|^{*}(N-\{\mid\}) \text {. }
$$

A one counter relation is a finite union of elementary one counter relations and the set of one counter relations is denoted by $\mathcal{C}$ ount.

For instance let $G$ be the one counter automaton defined by

$$
\begin{array}{ll}
\stackrel{a}{\longrightarrow}=\left.\right|^{*}(p, \mid p) \quad ; \quad \stackrel{b}{\longrightarrow}=\left.\left.\right|^{*}(\mid p, q) \cup\right|^{*}(\mid q, q) \\
\stackrel{\iota}{\longrightarrow}=\{(p, p)\} \quad ; \quad \stackrel{o}{\longrightarrow}=\{(p, p),(q, q)\}
\end{array}
$$

and represented as follows:

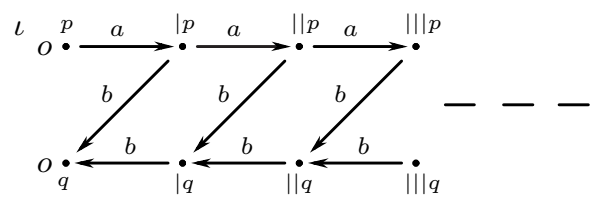

The (unique) morphism $f$ from $G$ into $\operatorname{Inp}_{1,1}(\{b\},\{c\},\{a\})$ is the one counter relation

$$
f=\left.\left.\right|^{*}(p, \kappa) \cup\right|^{*}(q, \kappa) .
$$

So $\left\{a^{n} b^{n} \mid n \geq 0\right\}=\mathrm{L}(\mathrm{G}) \in \operatorname{sRec}_{\mathcal{F} \text { (Count) }}\left(\operatorname{Inp}_{1,1}(\{\mathrm{~b}\},\{\mathrm{c}\},\{\mathrm{a}\})\right)$.

Like for finite automata, we need to determinize to get the closure under complementation by structural recognizability.

Definition 2. For $G \stackrel{f}{\longrightarrow} H$, we say that the morphism $f$ is deterministic and we write $G \stackrel{f}{\longrightarrow} H$ if the images by $f$ are distinct for distinct initial vertices, and for distinct goals of edges with the same source and label:

$$
\iota s, \iota t \in G \vee(r \underset{G}{\stackrel{a}{\longrightarrow}} s \wedge r \underset{G}{\stackrel{a}{\longrightarrow}} t) \Longrightarrow s=t \vee f(s) \neq f(t) \text {. }
$$

The reduction of a deterministic automaton is deterministic:

$$
(G \text { deterministic } \wedge G \stackrel{f}{\longrightarrow} H) \Longrightarrow G \stackrel{f}{\longrightarrow} H \text {. }
$$


The deterministic morphism preserves by inverse the determinism and the nonambiguity:

$(G \stackrel{f}{\longrightarrow} H \wedge H$ deterministic/unambiguous $) \Longrightarrow G$ deterministic/unambiguous.

Definition 3. For any relation family $\mathcal{R}$ and any automaton $H$, let

$$
\operatorname{dsRec}_{\mathcal{F}(\mathcal{R})}(H)=\{\mathrm{L}(G) \mid G \in \mathcal{F}(\mathcal{R}) \wedge \exists f \in \mathcal{R}(G \stackrel{f}{\longrightarrow} H)\}
$$

be the set of deterministic structural recognized languages.

Structural recognizability allows to get boolean algebras not only for pushdown or stack of stack automata but for each level of the stack hierarchy $[2,8]$ and also by allowing multi-stacks.

For any integers $m, n \geq 1$, we define the set $\mathcal{S} \operatorname{tack}(m, n)$ of relations definable by $m$ stacks of level $n$ and its subset $\mathcal{C} \operatorname{count}(m, n)$ of relations definable by $m$ counters of level $n$. This hierarchy of relation families is represented by the following figure:

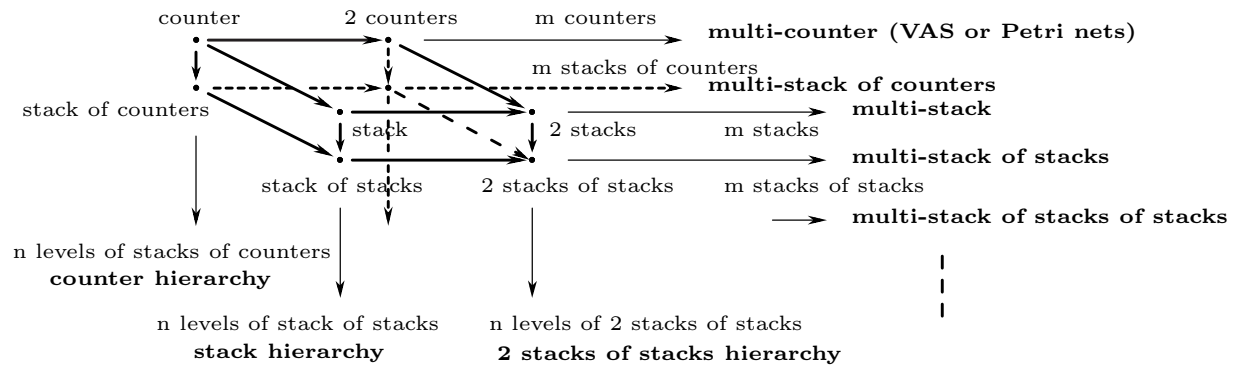

We have $\mathcal{C}$ ount $(1,1)=\mathcal{C}$ ount, $\mathcal{S} \operatorname{tack}(1,1)=\mathcal{S}$ tack and $\mathcal{S} \operatorname{tack}(1,2)=\mathcal{S}_{\text {tack }_{2}}$. For those families, structural recognizability and deterministic structural recognizability coincide and define a boolean algebra for each unambiguous automaton.

Theorem 2. For any $m, n>0$ and any unambiguous $H \in \mathcal{F}(\mathcal{S} \operatorname{tack}(m, n))$,

$$
\operatorname{sRec}_{\mathcal{F}(\mathcal{S} \operatorname{tack}(m, n))}(H)=\operatorname{dsRec}_{\mathcal{F}(\mathcal{S} \operatorname{tack}(m, n))}(H)
$$

is a boolean algebra with respect to $\mathrm{L}(H)$.

In fact, we have defined properties of stability on the automaton family $\mathcal{F}$ such that $\operatorname{sRec}_{\mathcal{F}}(H)=\operatorname{dsRec}_{\mathcal{F}}(H)$ is a boolean algebra for any unambiguous automaton $H$ in $\mathcal{F}$. We have proved that those stability properties are satisfied at each level of this multi-stack of stack hierarchy but more generally, structural recognizability can be applied to any stable automaton family. In the last section, we chose to apply it to synchronized automata. 


\section{Synchronized automata}

Synchronized automata can be of infinite degree or of finite but unbounded degree. They accept all the context-sensitive languages.

An elementary synchronized relation is of the form

$$
R .(U, V)=\left\{\left(w_{1} u, w_{2} v\right) \mid\left(w_{1}, w_{2}\right) \in R, u \in U, v \in V\right\}
$$

where $R \in \operatorname{Reg}\left((N \times N)^{*}\right)$ is a letter-to-letter regular relation and $U, V \in$ $\operatorname{Reg}\left(N^{*}\right)$ are regular sets. A synchronized relation is a finite union of elementary synchronized relations and the set of synchronized relations is denoted by $\mathcal{S} y n c$. For instance, the automaton $H_{2}$ defined by

$$
\begin{array}{ll}
\stackrel{a}{\longrightarrow}=(A, A)^{*}(B, A)(\varepsilon, B) & ; \quad \stackrel{b}{\longrightarrow}=(B, B)(A, A)^{*}(A, \varepsilon) \\
\stackrel{c}{\longrightarrow}=\left[(A, A)^{*}(A, B)(B, A)(A, A)^{*}\right](\varepsilon, \epsilon) & ; \stackrel{\iota}{\longrightarrow}=\stackrel{o}{\longrightarrow}=(B, B)(\varepsilon, \epsilon)
\end{array}
$$

belongs to $\mathcal{F}\left(\mathcal{S}_{\text {ync }}\right)$ and is represented below.

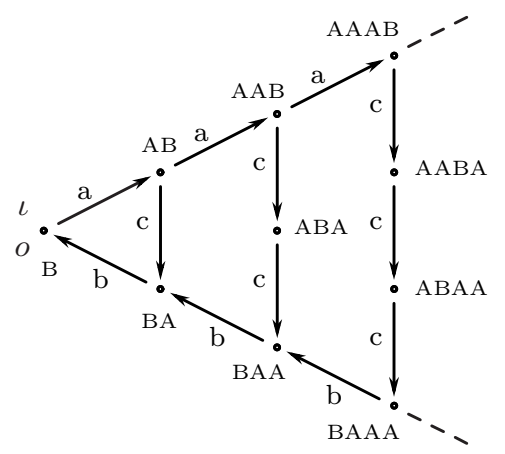

For the family $\mathcal{F}(\mathcal{S}$ ync) of synchronized automata, the deterministic structural recognizability gives a boolean algebra for each unambiguous synchronized automaton.

Theorem 3. For any unambiguous automaton $H \in \mathcal{F}(\mathcal{S} y n c)$,

$\operatorname{dsRec}_{\mathcal{F}\left(\mathcal{S}_{\mathrm{ync}}\right)}(H)$ is a boolean algebra with respect to $\mathrm{L}(H)$.

The languages accepted by automata of $\mathcal{F}(\mathcal{S}$ ync $)$ are the context-sensitive languages [14]. Given an alphabet $T$, any synchronized automaton $G$ labelled in $T$ is reducible to $\operatorname{Loop}_{T}$ by the morphism mapping all vertices of $V_{G}$ to the unique vertex $\kappa$ of $\operatorname{Loop}_{T}$. So

$\operatorname{sRec}_{\mathcal{F}\left(\mathcal{S}_{\text {ync }}\right)}\left(\operatorname{Loop}_{T}\right)$ is the set of context-sensitive languages over $T$

which is a boolean algebra $[9,16]$. Furthermore $\operatorname{dsRec}_{\mathcal{F}\left(\mathcal{S}_{\text {ync }}\right)}\left(\operatorname{Loop}_{T}\right)$ is the set of deterministic context-sensitive languages over $T$ which is a boolean algebra by Theorem 3. Thus, the following question arises:

$$
\operatorname{sRec}_{\mathcal{F}(\mathcal{S} y n c)}\left(\operatorname{Loop}_{T}\right)=\operatorname{dsRec}_{\mathcal{F}(\mathcal{S} y n c)}\left(\operatorname{Loop}_{T}\right) ?
$$

This corresponds to the famous conjecture of Kuroda [10].

A more general question is:

$\operatorname{sRec}_{\mathcal{F}\left(\mathcal{S}_{\text {ync })}\right.}(H)=\operatorname{dsRec}_{\mathcal{F}\left(\mathcal{S}_{y n c}\right)}(H)$ for any unambiguous $H \in \mathcal{F}\left(\mathcal{S}_{\text {ync }}\right)$ ? 
For a given partition $\vec{T}=\left(T_{-1}, T_{0}, T_{1}\right)$, let us also characterize by structural recognizability the family

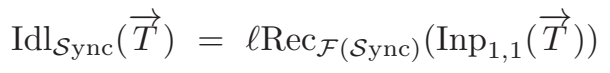

of input-driven synchronized languages. For instance, for the previous automaton $\mathrm{H}_{2}$, we have

$$
\mathrm{L}\left(H_{2}\right)=\left\{a^{n} c^{n} b^{n} \mid n \geq 0\right\}^{*} \in \operatorname{Idl}_{\text {Sync }}(\{b\},\{c\},\{a\}) .
$$

For a given partition $\vec{T}$ with $T_{-1}, T_{1} \neq \emptyset$,

$$
\operatorname{Idl}_{S \text { ync }}(\vec{T}) \subset \operatorname{sRec}_{\mathcal{F}(S \text { ync })}\left(\operatorname{Inp}_{1,1}(\vec{T})\right) .
$$

For instance $\operatorname{Inp}_{1,1}(\{d\}, \emptyset,\{c\})$ can be reduced to $\operatorname{Inp}_{1,1}(\{b\},\{c, d\},\{a\})$ so the language $\mathrm{L}\left(\operatorname{Inp}_{1,1}(\{d\}, \emptyset,\{c\})\right)$ belongs to $\operatorname{sRec}_{\mathcal{F}\left(\mathcal{S}_{y n c}\right)}\left(\operatorname{Inp}_{1,1}(\{b\},\{c, d\},\{a\})\right)$ but this language is not an input-driven synchronized language for the partition $(\{b\},\{c, d\},\{a\})$ : all the vertices of $\operatorname{Inp}_{1,1}(\{d\}, \emptyset,\{c\})$ are mapped to the same vertex $\kappa$ and it is not possible to have an infinite number of vertices of the same length.

To characterize $\operatorname{Idl}_{\mathcal{S} \text { ync }}(\vec{T})$, we restrict to the subfamily of synchronized relations $R$ of bounded length difference:

$$
\exists b, \forall(u, v) \in R,|| u|-| v|| \leq b .
$$

So a synchronized relation of bounded length difference is a finite union of relations of the form

$$
R .(u, v)=\left\{\left(w_{1} u, w_{2} v\right) \mid\left(w_{1}, w_{2}\right) \in R\right\}
$$

where $R \in \operatorname{Reg}\left((N \times N)^{*}\right)$ is a letter-to-letter regular relation and $u, v \in N^{*}$. Denoting by bSync the set of synchronized relations of bounded length difference, the bounded length difference synchronized automata of $\mathcal{F}(\mathrm{b} \mathcal{S}$ ync) recognize the same languages as synchronized automata of $\mathcal{F}(\mathcal{S}$ ync):

$\mathcal{L}(\mathcal{F}$ (bS ync $))$ is the family of context-sensitive languages [14].

This restriction to $\mathcal{F}$ (bS $\mathcal{S y n c}$ ) allows to characterize by structural recognizability the family $\operatorname{Idl}_{\mathcal{S} \text { ync }}(\vec{T})=\operatorname{Idl}_{\text {b Sync }}(\vec{T})$ of input-driven synchronized languages.

Proposition 5.1 For any partition $\vec{T}$,

$$
\operatorname{sRec}_{\mathcal{F}(b S \text { ync })}\left(\operatorname{Inp}_{1,1}(\vec{T})\right)=\operatorname{Idl}_{\text {Sync }}(\vec{T}) .
$$

However we do not know whether $\operatorname{Idl}_{\text {Sync }}(\vec{T})$ is a boolean algebra with respect to $\mathrm{L}\left(\operatorname{Inp}_{1,1}(\vec{T})\right)$. This is true when restricting to $\operatorname{dsRec}_{\mathcal{F}(b S \mathrm{Sync})}\left(\operatorname{Inp}_{1,1}(\vec{T})\right)$.

Theorem 4. For any unambiguous automaton $H \in \mathcal{F}(\mathcal{S} y n c)$, $\operatorname{dsRec}_{\mathcal{F} \text { (bSync) }}(H)$ is a boolean algebra with respect to $\mathrm{L}(H)$.

Theorems 2,3 and 4 are obtained in a same general way: for an automaton family $\mathcal{F}(\mathcal{R})$, we define a condition on the relation family $\mathcal{R}$ over the vertices. This condition ensures that vertices can be added or deleted while staying in the same automaton family. It allows to make standard constructions on automata such as determinization, synchronization product and complementation restricted to vertices having the same morphic image. 


\section{Conclusion}

We have defined structural recognizability by inverse morphism for infinite automata. We have applied it to automata of the multi-stack of stack hierarchy and to synchronized automata and proved that, in those cases, any unambiguous automaton defines a boolean algebra of languages.

In fact, structural recognizability can be applied to any unambiguous recognizer and any stable automaton family.

Thanks to Arnaud Carayol for his remarks and for the example of Proposition 3.1.

\section{References}

1. A. Aho Indexed grammar-an extension of context-free grammars, Journal of the ACM 15 (4): 647-671 (1968).

2. A. Carayol Regular sets of higher-order pushdown stacks, $30^{\text {th }}$ MFCS J. Jȩdrzejowicz and A. Szepietowski (Eds.), LNCS 3618, 168-179 (2005).

3. D. Caucal On infinite transition graphs having a decidable monadic theory, Theoretical Computer Science 290, 79-115 (2003).

4. D. Caucal Boolean algebras of unambiguous context-free languages, $28^{\text {th }}$ FSTTCS, Dagstuhl Research Online Publication Server, R. Hariharan, M. Mukund, V. Vinay (Eds.) (2008).

5. S. Eilenberg, Algèbre catégorique et théorie des automates, Institut H. Poincaré, Université de Paris (1967).

6. S. Eilenberg, Automata, languages and machines, Vol. A, Academic Press, NewYork (1974).

7. C. Elgot and J. Mezei, On relations defined by generalized finite automata, IBM Journal of Research and Development 9(1), 47-68 (1965).

8. S. Fratani, Automates à piles de piles ... de piles, $\mathrm{PhD}$ thesis, University Bordeaux 1 (2005).

9. N. Immerman, Nondeterministic space is closed under complementation, SIAM J. Comput., 17(5), 935-938 (1988).

10. S. Kuroda, Classes of languages and linear-bounded automata., Information and control, 7(2), 207-223 (1964).

11. A. Maslov, The hierarchy of indexed languages of an arbitrary level, Doklady Akademii Nauk SSSR 217, 1013-1016 (1974).

12. K. Mehlhorn, Pebbling mountain ranges and its application to DCFL recognition, $7^{\text {th }}$ ICALP, LNCS 85, J. de Bakker, J. van Leeuwen (Eds.), 422-432 (1980).

13. D. Nowotka and J. Srba, Height-deterministic pushdown automata, $32^{\text {nd }}$ MFCS, LNCS 4708, L. Kucera, A. Kucera (Eds.), 125-134 (2007).

14. C. Rispal, The synchronized graphs trace the context-sensitive languages. Electr. Notes Theor. Comput. Sci. 68(6), 55-70 (2002)

15. A. Semenov, Decidability of monadic theories, $11^{\text {th }}$ MFCS, LNCS 176, M. Chytil, V. Koubek (Eds.), 162-175 (1984).

16. R. Szelepcsnyi, The method of forced enumeration for nondeterministic automata, Acta Informatica 26(3), 279-284 (1988).

17. W. Thomas, Uniform and nonuniform recognizability, Theoretical Computer Science 292, 299-316 (2003). 


\section{Appendix}

We give here proofs and complementary results.

\section{A Operations on automata}

The closure of $\operatorname{sRec}_{\mathcal{F}(\mathcal{R})}(H)$ under complementation and intersection are obtained by closure of the automaton family

$$
\operatorname{Aut}_{\mathcal{F}(\mathcal{R})}(H)=\{G \in \mathcal{F}(\mathcal{R}) \mid \exists f \in \mathcal{R}(G \stackrel{f}{\longrightarrow} H)\}
$$

under standard determinization and synchronization product but restricted to vertices reducible to a same vertex.

\section{A.a Determinization}

An automaton is reduced if any vertex is accessible from an initial vertex and coaccessible from a final vertex. Let us consider a reduction $G \stackrel{f}{\longrightarrow} H$ from a reduced automaton $G$ into a unambiguous automaton $H$. This morphism $f$ is unique.

Proposition A.1 There is at most one morphism from a reduced automaton into a unambiguous automaton.

\section{Proof.}

Let $G \stackrel{g}{\longrightarrow} H$ and $G \stackrel{h}{\longrightarrow} H$ with $G$ reduced and $H$ unambiguous.

Let $s$ be any vertex of $G$.

As $G$ is reduced, there exists $u, v$ such that $\iota \stackrel{\vec{G}}{\Rightarrow} s \underset{G}{\stackrel{v}{\Longrightarrow}} o$ meaning that

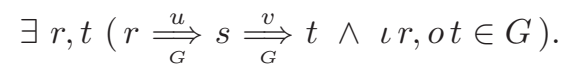

As $g$ and $h$ are morphisms, we have

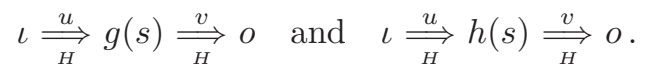

As $H$ is unambiguous, $g(s)=h(s)$.

We partially determinize $G$ by applying the standard powerset construction restricted to the subsets of vertices having the same image by $f$.

Precisely and for any $a \in \mathrm{T}_{G} \cup\{\iota, o\}$, the image by $\underset{G}{\stackrel{a}{\rightarrow}}$ of $P \subseteq \mathrm{V}_{G}$ is

$$
\underset{G}{\stackrel{a}{\longrightarrow}}(P)=\{t \mid \exists s \in P(s \underset{G}{\stackrel{a}{\rightarrow}} t)\}
$$

the set of goals of $a$-edges of sources in $P$. In particular for $c \in\{\iota, o\}$,

$$
\underset{G}{\stackrel{c}{c}}(P)=\{s \in P \mid c s \in G\}
$$

is the set of vertices in $P$ coloured by $c$. 
Let $\Pi$ be the family of non empty subsets of $\mathrm{V}_{G}$ with the same image by $f$ :

$$
\Pi=\left\{P \subseteq \mathrm{V}_{G}|| f(P) \mid=1\right\} .
$$

The determinization $\operatorname{Det}(G, f)$ of $G$ according to $f$ is the following automaton:

$$
\begin{aligned}
\operatorname{Det}(G, f) & =\{\iota P \mid P \in \Pi \wedge \forall \bar{P} \in \Pi(\bar{P} \supseteq P \Longrightarrow \underset{\rightarrow}{\longrightarrow}(\bar{P})=P)\} \\
& \cup\{P \stackrel{a}{\longrightarrow} Q \mid P, Q \in \Pi \wedge \forall \bar{Q} \in \Pi(\bar{Q} \supseteq Q \Longrightarrow \underset{G}{\longrightarrow}(P) \cap \bar{Q}=Q)\} \\
& \cup\{o P \mid P \in \Pi \wedge \underset{G}{\stackrel{o}{G}}(P) \neq \emptyset\} .
\end{aligned}
$$

By extending $f: \Pi \longrightarrow \mathrm{V}_{H}$ by $f(P)=f(s)$ for any $s \in P \in \Pi$, we get

$$
\operatorname{Det}(G, f) \stackrel{f}{\longrightarrow} H \text {. }
$$

The automaton $\operatorname{Det}(G, f)$ is deterministic according to $f$ in the following sense:

$$
\begin{aligned}
& G \stackrel{f}{\longrightarrow} H \text { is a deterministic reduction and we write } G \stackrel{f}{\longrightarrow} H \text { if } \\
& \qquad s, \iota t \in G \vee(r \underset{G}{\longrightarrow} s \wedge r \underset{G}{\longrightarrow} t) \Longrightarrow s=t \vee f(s) \neq f(t)
\end{aligned}
$$

meaning that the images by $f$ are distinct for initial vertices and for goals of arcs with the same source and label.

Lemma A.2 For $G \stackrel{f}{\longrightarrow} H$, we have

$$
\operatorname{Det}(G, f) \stackrel{f}{\rightarrow} H \text { and } \mathrm{L}(\operatorname{Det}(G, f))=\mathrm{L}(G) .
$$

\section{Proof.}

i) Let us show that $\mathrm{L}(\operatorname{Det}(G, f))=\mathrm{L}(G)$.

$\subseteq$ : Let $a_{1} \ldots a_{n} \in \mathrm{L}(\operatorname{Det}(G, f))$ with $n \geq 0$ and $a_{1}, \ldots, a_{n} \in T$.

There exists $P_{0} \underset{\operatorname{Det}(G, f)}{\stackrel{a_{1}}{\longrightarrow}} P_{1} \ldots \underset{\operatorname{Det}(G, f)}{\stackrel{a_{n}}{\longrightarrow}} P_{n}$ with $\iota P_{0}, o P_{n} \in \operatorname{Det}(G, f)$.

There is $s_{n} \in P_{n}$ such that $o s_{n} \in G$.

By induction from $i=n$ to $i=1$,

$$
\text { there exists } s_{i-1} \in P_{i-1} \text { such that } s_{i-1} \underset{G}{\frac{a_{i}}{G}} s_{i} \text {. }
$$

As $s_{0} \in P_{0}$, we have $\iota s_{0} \in G$.

Finally $s_{0} \underset{G}{\stackrel{a_{1}}{\longrightarrow}} s_{1} \ldots \underset{G}{\stackrel{a_{n}}{\longrightarrow}} s_{n}$ with $\iota s_{0}, o s_{n} \in G$ hence $a_{1} \ldots a_{n} \in \mathrm{L}(G)$.

$\supseteq$ : Let $a_{1} \ldots a_{n} \in \mathrm{L}(G)$ with $n \geq 0$ and $a_{1}, \ldots, a_{n} \in T$.

There exists $s_{0} \underset{G}{\stackrel{a_{1}}{\longrightarrow}} s_{1} \ldots \underset{G}{\stackrel{a_{n}}{\longrightarrow}} s_{n}$ with $\iota s_{0}, o s_{n} \in G$.

We take the maximal (for inclusion) subset $P_{0} \in \Pi$ such that

$$
\text { for any } s \in P_{0}, \iota s \in G \wedge f(s)=f\left(s_{0}\right) \text {. }
$$

In particular $s_{0} \in P_{0}$ and $\iota P_{0} \in \operatorname{Det}(G, f)$.

By induction on $1 \leq i \leq n$, we take the maximal subset $P_{i} \in \Pi$ such that

$$
P_{i} \subseteq \underset{G}{\stackrel{a_{i}}{\longrightarrow}}\left(P_{i-1}\right) \text { and } f(s)=f\left(s_{i}\right) \text { for any } s \in P_{i} ;
$$

in particular $s_{i} \in P_{i}$ and $P_{i-1} \underset{\operatorname{Det}(G, f)}{\stackrel{a_{i}}{\longrightarrow}} P_{i}$.

As $s_{n} \in P_{n}$ we have $o P_{n} \in \operatorname{Det}(G, f)$.

Thus $P_{0} \underset{\operatorname{Det}(G, f)}{\stackrel{a_{1}}{\longrightarrow}} P_{1} \ldots \underset{\operatorname{Det}(G, f)}{\stackrel{a_{n}}{\longrightarrow}} P_{n}$ with $\iota P_{0}, o P_{n} \in \operatorname{Det}(G, f)$.

Hence $a_{1} \ldots a_{n} \in \mathrm{L}(\operatorname{Det}(G, f))$.

ii) It remains to check that $\operatorname{Det}(G, f) \stackrel{f}{\longrightarrow} H$. 
First $\operatorname{Det}(G, f)$ is $f$-deterministic since for

$$
\left(\iota Q, \iota Q^{\prime} \in \operatorname{Det}(G, f) \vee\left(P \underset{\operatorname{Det}(G, f)}{\stackrel{a}{\longrightarrow}} Q \wedge P \underset{\operatorname{Det}(G, f)}{\stackrel{a}{\longrightarrow}} Q^{\prime}\right)\right) \text { with } f(Q)=f\left(Q^{\prime}\right)
$$

we have $Q \cup Q^{\prime} \in \Pi$ hence by maximality of $Q$ and $Q^{\prime}$, we get $Q=Q^{\prime}$.

Let $c P \in \operatorname{Det}(G, f)$ with $c \in\{\iota, o\}$.

There is $s \in P$ such that $c s \in G$.

So $c f(s) \in H$ and $f(s)=f(P)$ i.e. $c f(P) \in H$.

Let $P \underset{\operatorname{Det}(G, f)}{\stackrel{a}{\longrightarrow}} Q$. So $Q \neq \emptyset$ and we take $t \in Q$.

As $Q \subseteq \underset{G}{\stackrel{a}{\rightarrow}}(P)$, there is $s \in P$ such that $s \underset{G}{\stackrel{a}{\longrightarrow}} t$.

Thus $f(P)=f(s) \underset{H}{\stackrel{a}{\longrightarrow}} f(t)=f(Q)$.

The deterministic reduction preserves determinism and unambiguity by inverse.

Lemma A.3 Let $G \stackrel{f}{\longrightarrow} H$.

If $H$ is deterministic then $G$ is deterministic.

If $H$ is unambiguous then $G$ is unambiguous and

$$
(s \stackrel{u}{\Longrightarrow} t, \iota s \in G, u \in \mathrm{L}(G), o f(t) \in H) \Longrightarrow o t \in G \text {. }
$$

\section{Proof.}

i) Let $G \stackrel{f}{\longrightarrow} H$ with $H$ deterministic. Let us check that $G$ is deterministic.

Let $\iota s, \iota t \in G$. As $f$ is a morphism, $\iota f(s), \iota f(t) \in H$.

As $H$ is deterministic, we have $f(s)=f(t)$.

As $G$ is $f$-deterministic, we get $s=t$.

Let $r \underset{G}{\stackrel{a}{\longrightarrow}} s$ and $r \underset{G}{\stackrel{a}{\longrightarrow}} t$.

As $f$ is a morphism, $f(r) \underset{G}{\stackrel{a}{\longrightarrow}} f(s)$ and $f(r) \underset{G}{\stackrel{a}{\longrightarrow}} f(t)$.

As $H$ is deterministic, we have $f(s)=f(t)$ hence $s=t$.

ii) Let $G \stackrel{f}{\longrightarrow} H$ with $H$ unambiguous. Let us check that $G$ is unambiguous. Let $s_{0} \underset{G}{\stackrel{a_{1}}{\rightarrow}} s_{1} \ldots \stackrel{a_{n}}{\rightarrow} s_{n}$ and $t_{0} \underset{G}{\stackrel{a_{1}}{\rightarrow}} t_{1} \ldots \underset{G}{\stackrel{a_{n}}{G}} t_{n}$ with $\iota s_{0}, \iota t_{0}, o s_{n}, o t_{n} \in G$.

As $f$ is a morphism, we have

$$
f\left(s_{0}\right) \underset{H}{\stackrel{a_{1}}{\longrightarrow}} f\left(s_{1}\right) \ldots \underset{H}{\stackrel{a_{n}}{\longrightarrow}} f\left(s_{n}\right) \text { and } f\left(t_{0}\right) \underset{H}{\stackrel{a_{1}}{\longrightarrow}} f\left(t_{1}\right) \ldots \underset{H}{\stackrel{a_{n}}{\longrightarrow}} f\left(t_{n}\right)
$$

with $\iota f\left(s_{0}\right), \iota f\left(t_{0}\right), o f\left(s_{n}\right), o f\left(t_{n}\right) \in H$.

As $H$ is unambiguous, $f\left(s_{0}\right)=f\left(t_{0}\right), \ldots, f\left(s_{n}\right)=f\left(t_{n}\right)$.

As $G$ is $f$-deterministic and by induction on $0 \leq i \leq n$, we get $s_{i}=t_{i}$.

Thus $G$ is unambiguous.

iii) Let $s \stackrel{a_{1} \ldots a_{n}}{\Rightarrow} t$ with $n \geq 0, \iota s \in G, a_{1} \ldots a_{n} \in \mathrm{L}(G)$ and $o f(t) \in H$.

Let us check that $o t \in G$.

There is $s_{0} \stackrel{a_{1}}{\longrightarrow} s_{1} \ldots \stackrel{a_{n}}{\longrightarrow} s_{n}$ with $s_{0}=s$ and $s_{n}=t$.

As $a_{1} \ldots a_{n} \in \mathrm{L}(G)$, there exists $t_{0} \underset{G}{\stackrel{a_{1}}{\longrightarrow}} t_{1} \ldots \underset{G}{\stackrel{a_{n}}{\rightarrow}} t_{n}$ with $\iota t_{0}, o t_{n} \in G$.

As $f$ is a morphism, 


$$
f\left(s_{0}\right) \underset{H}{\stackrel{a_{1}}{\longrightarrow}} f\left(s_{1}\right) \ldots \underset{H}{\stackrel{a_{n}}{\longrightarrow}} f\left(s_{n}\right) \text { and } f\left(t_{0}\right) \underset{H}{\stackrel{a_{1}}{\longrightarrow}} f\left(t_{1}\right) \ldots \underset{H}{\stackrel{a_{n}}{\longrightarrow}} f\left(t_{n}\right)
$$

with $\iota f\left(s_{0}\right), \iota f\left(t_{0}\right), o f\left(s_{n}\right), o f\left(t_{n}\right) \in H$.

As $H$ is unambiguous, $f\left(s_{0}\right)=f\left(t_{0}\right), \ldots, f\left(s_{n}\right)=f\left(t_{n}\right)$.

As $G$ is $f$-deterministic, we get $s_{0}=t_{0}, \ldots, s_{n}=t_{n}$. Thus ot $=o t_{n} \in G$.

\section{A.b Inversion}

Let us consider a reduction $G \stackrel{f}{\longrightarrow} H$ between disjoint automata: $\mathrm{V}_{G} \cap \mathrm{V}_{H}=\emptyset$. Let us define an automaton reducible to $H$, and accepting $\mathrm{L}(H)-\mathrm{L}(G)$ for $H$ unambiguous. The inverse automaton is the following automaton:

$$
\begin{aligned}
\operatorname{Inv}(G, f, H) & =\left(G-o \mathrm{~V}_{G}\right) \cup\{o s \notin G \mid o f(s) \in H\} \\
& \cup H-\{\iota f(s) \mid \iota s \in G\} \\
& \cup\{s \stackrel{a}{\longrightarrow} q \mid f(s) \underset{H}{\stackrel{a}{\longrightarrow}} q \wedge \neg \exists t(s \underset{G}{\stackrel{a}{\longrightarrow}} t \wedge f(t)=q)\} .
\end{aligned}
$$

This inversion is illustrated below (without specifying the vertices and the unique morphism).
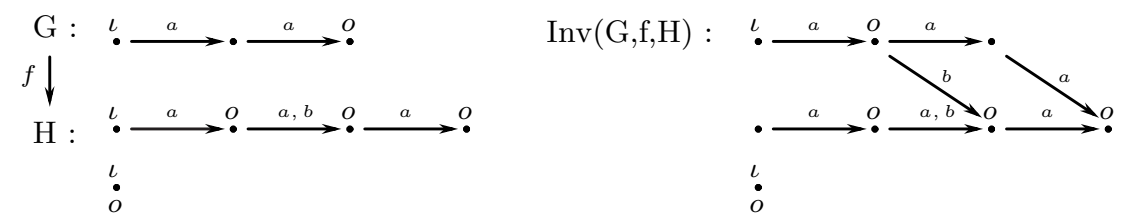

In this example, $\mathrm{L}(\operatorname{Inv}(G, f, H))=\{\varepsilon, a, a b, a a a, a b a\}=\mathrm{L}(H)-\mathrm{L}(G)$.

The automaton $\operatorname{Inv}(G, f, H)$ recognizes $\mathrm{L}(H)-\mathrm{L}(G)$ when $G \stackrel{f}{\longrightarrow} H$ is a deterministic reduction and $H$ is unambiguous.

Lemma A.4 For $\mathrm{V}_{G} \cap \mathrm{V}_{H}=\emptyset$, we have

$$
\begin{aligned}
& G \stackrel{f}{\longrightarrow} H \Longrightarrow \operatorname{Inv}(G, f, H) \stackrel{f \cup \operatorname{Id}_{H}}{\longrightarrow} H ; \\
& G \stackrel{f}{\longrightarrow} H \quad \Longrightarrow \operatorname{Inv}(G, f, H) \stackrel{f \cup \operatorname{Id}_{H}}{\longrightarrow} H \\
& \text { and } \quad \mathrm{L}(\operatorname{Inv}(G, f, H))=\mathrm{L}(H)-\mathrm{L}(G) \text { for } H \text { unambiguous. }
\end{aligned}
$$

Proof.

i) Let $G \stackrel{f}{\longrightarrow} H$ and $f_{H}=f \cup \operatorname{Id}_{H}$. Let us check that $\operatorname{Inv}(G, f, H) \stackrel{f_{H}}{\longrightarrow} H$.

Let $s \underset{\operatorname{Inv}(G, f, H)}{\stackrel{a}{\longrightarrow}} t$. We have the three complementary cases below.

Case 1:s, $t \in \mathrm{V}_{G}$.

So $s \underset{G}{\stackrel{a}{\longrightarrow}} t$ hence $f_{H}(s)=f(s) \underset{H}{\stackrel{a}{\longrightarrow}} f(t)=f_{H}(t)$.

Case 2: $s, t \in \mathrm{V}_{H}$.

So $s \underset{H}{\stackrel{a}{\longrightarrow}} t$ hence $f_{H}(s)=s \underset{H}{\stackrel{a}{\longrightarrow}} t=f_{H}(t)$.

Case $3: s \in \mathrm{V}_{G}$ and $t \in \mathrm{V}_{H}$. 
So $f(s) \underset{H}{\stackrel{a}{\longrightarrow}} t$. Thus $f_{H}(s)=f(s) \underset{H}{\stackrel{a}{\longrightarrow}} t=f_{H}(t)$.

Let $c s \in \operatorname{Inv}(G, f, H)$ with $c \in\{\iota, o\}$.

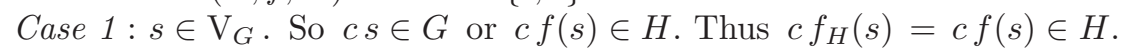

Case 2: $s \in \mathrm{V}_{H}$. So $c s \in H$ hence $c f_{H}(s)=c s \in H$.

ii) Let $G \stackrel{f}{\longrightarrow} H$. Let us show that $\operatorname{Inv}(G, f, H) \stackrel{f_{H}}{\longrightarrow} H$.

By (i), it remains to check that $\operatorname{Inv}(G, f, H)$ is $f_{H}$-deterministic.

Let $\iota s, \iota t \in \operatorname{Inv}(G, f, H)$ with $f_{H}(s)=f_{H}(t)$.

We have the complementary cases below.

Case $1: s, t \in \mathrm{V}_{G}$.

So $\iota s, \iota t \in G$ and $f(s)=f_{H}(s)=f_{H}(t)=f(t)$.

As $G$ is $f$-deterministic, $s=t$.

Case 2: $s \in \mathrm{V}_{G}$ and $t \in \mathrm{V}_{H}$.

So $\iota s \in G$, $\iota t \in H$ and $\forall r(f(r)=t \Longrightarrow \iota r \notin G)$.

Furthermore $f(s)=f_{H}(s)=f_{H}(t)=t$.

Thus $\iota s \notin G$. This contradiction means that Case 2 is not possible.

Case 3: $s \in \mathrm{V}_{H}$ and $t \in \mathrm{V}_{G}$.

This case is symmetric to Case 2 hence is not possible.

Case $4: s, t \in \mathrm{V}_{H}$.

So $\iota s, \iota t \in H$ and $s=f_{H}(s)=f_{H}(t)=t$.

Let $r \underset{\operatorname{Inv}(G, f, H)}{\stackrel{a}{\longrightarrow}} s$ and $r \underset{\operatorname{Inv}(\overrightarrow{G, f, H)}}{\stackrel{a}{\longrightarrow}} t$ with $f_{H}(s)=f_{H}(t)$.

We have $s \in V_{G} \Longleftrightarrow t \in V_{G}$.

In fact, assume that $s \in \mathrm{V}_{G}$ and $t \in \mathrm{V}_{H}$.

So $r \underset{G}{\stackrel{a}{\longrightarrow}} s$ and $f(s)=f_{H}(s)=f_{H}(t)=t$.

As $r \underset{\operatorname{Inv}(G, f, H)}{\stackrel{a}{\longrightarrow}} t$ with $r \underset{G}{\stackrel{a}{\longrightarrow}} s$ and $f(s)=t$,

we have a contradiction by definition of $\operatorname{Inv}(G, f, H)$.

It remains the two cases below.

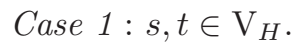

So $s=f_{H}(s)=f_{H}(t)=t$.

Case 2: $s, t \in \mathrm{V}_{G}$.

So $r \stackrel{a}{\longrightarrow} s$ and $r \stackrel{a}{\longrightarrow} t$.

Furthermore $f(s)=f_{H}(s)=f_{H}(t)=f(t)$.

As $G$ is $f$-deterministic, we get $s=t$.

iii) Let $G \stackrel{f}{\rightarrow} H$ with $H$ unambiguous.

Let us show that $\mathrm{L}(\operatorname{Inv}(G, f, H))=\mathrm{L}(H)-\mathrm{L}(G)$.

$\supseteq$ : Let $a_{1} \ldots a_{n} \in \mathrm{L}(H)-\mathrm{L}(G)$ for some $n \geq 0$ and $a_{1}, \ldots, a_{n} \in T$.

There is $t_{0} \underset{H}{\stackrel{a_{1}}{\longrightarrow}} t_{1} \ldots \underset{H}{a_{n}} t_{n}$ with $\iota t_{0}, o t_{n} \in H$.

Case 1 : There is no $s \in V_{G}$ such that $\iota s \in G$ and $f(s)=t_{0}$.

So $\iota t_{0} \in \operatorname{Inv}(G, f, H)$.

We have $t_{0} \underset{\operatorname{Inv}(G, f, H)}{\stackrel{a_{1}}{\longrightarrow}} t_{1} \ldots \underset{\operatorname{Inv}(G, f, H)}{\stackrel{a_{n}}{\longrightarrow}} t_{n}$ with $\iota t_{0}, o t_{n} \in \operatorname{Inv}(G, f, H)$.

Hence $a_{1} \ldots a_{n} \in \mathrm{L}(\operatorname{Inv}(G, f, H))$.

Case 2: There is $s_{0}$ such that $\iota s_{0} \in G$ and $f\left(s_{0}\right)=t_{0}$. 
We take a maximal derivation $s_{0} \underset{G}{\stackrel{a_{1}}{\longrightarrow}} s_{1} \ldots \underset{G}{\stackrel{a_{m}}{\longrightarrow}} s_{m}$ such that $0 \leq m \leq n$ and $f\left(s_{1}\right)=t_{1}, \ldots, f\left(s_{m}\right)=t_{m}$.

Thus $s_{0} \underset{\operatorname{Inv}(G, f, H)}{\stackrel{a_{1}}{\longrightarrow}} s_{1} \ldots \underset{\operatorname{Inv}(G, f, H)}{\stackrel{a_{m}}{\longrightarrow}} s_{m}$ with $\iota s_{0} \in \operatorname{Inv}(G, f, H)$.

Case 2.1: $m=n$.

As $a_{1} \ldots a_{n} \notin \mathrm{L}(G), o s_{n} \notin G$. Furthermore $o f\left(s_{n}\right)=o t_{n} \in H$.

So $o s_{n} \in \operatorname{Inv}(G, f, H)$ hence $a_{1} \ldots a_{n} \in \mathrm{L}(\operatorname{Inv}(G, f, H))$.

Case 2.2: $m<n$.

By maximality of $m$, we have $s_{m} \underset{\operatorname{Inv}(G, f, H)}{\stackrel{a_{m+1}}{\longrightarrow}} t_{m+1}$.

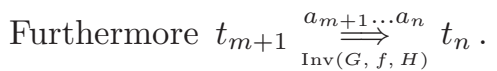

As $o t_{n} \in \operatorname{Inv}(G, f, H)$, we get $a_{1} \ldots a_{n} \in \mathrm{L}(\operatorname{Inv}(G, f, H))$.

$\subseteq$ : Let $u \in \mathrm{L}(\operatorname{Inv}(G, f, H))$. By (i), $u \in \mathrm{L}(H)$.

Assume that $u \in \mathrm{L}(G)$. There exists $s, t$ such that $s \underset{G}{\stackrel{u}{\longrightarrow}} t$ with $\iota s, o t \in G$.

So $s \underset{\operatorname{Inv}(G, f, H)}{\stackrel{u}{\longrightarrow}} t$ with $\iota s \in \operatorname{Inv}(G, f, H)$ and $o f(t) \in H$.

As $\operatorname{Inv}(G, f, H) \stackrel{f \cup \operatorname{Id}_{H}}{\longrightarrow} H$ unambiguous and by Lemma A.3, ot $\in \operatorname{Inv}(G, f, H)$.

This is a contradiction by definition of $\operatorname{Inv}(G, f, H)$.

\section{A.c Synchronization product}

Let us consider two reductions $G \stackrel{f}{\longrightarrow} H$ and $G^{\prime} \stackrel{f^{\prime}}{\longrightarrow} H$.

We partially synchronize $G$ and $G^{\prime}$ by applying standard synchronization product restricted to couple of vertices having the same morphic image. Precisely and for

$$
\Delta=\left\{\left(s, s^{\prime}\right) \in \mathrm{V}_{G} \times \mathrm{V}_{G^{\prime}} \mid f(s)=f^{\prime}\left(s^{\prime}\right)\right\}
$$

we define the mapping

$$
f \times f^{\prime}: \Delta \longrightarrow \mathrm{V}_{H} \text { by }\left(f \times f^{\prime}\right)\left(s, s^{\prime}\right)=f(s) \text { for any }\left(s, s^{\prime}\right) \in \Delta
$$

and we define the synchronization product

$$
\begin{aligned}
G \times f, f^{\prime} G^{\prime} & =\left\{\left(s, s^{\prime}\right) \stackrel{a}{\longrightarrow}\left(t, t^{\prime}\right) \mid s \stackrel{a}{\longrightarrow} t \wedge s^{\prime} \stackrel{a}{\longrightarrow}_{G^{\prime}} t^{\prime} \wedge\left(s, s^{\prime}\right),\left(t, t^{\prime}\right) \in \Delta\right\} \\
& \cup\left\{c\left(s, s^{\prime}\right) \mid c \in\{\iota, o\} \wedge c s \in G \wedge c s^{\prime} \in G^{\prime} \wedge\left(s, s^{\prime}\right) \in \Delta\right\} .
\end{aligned}
$$

The automaton $G \times f, f^{\prime} G^{\prime}$ recognizes $\mathrm{L}(G) \cap \mathrm{L}\left(G^{\prime}\right)$ for $H$ unambiguous.

$$
\begin{aligned}
& \text { Lemma A.5 We have } \\
& G \stackrel{f}{\longrightarrow} H \wedge G^{\prime} \stackrel{f^{\prime}}{\longrightarrow} H \Longrightarrow G \times{ }_{f, f^{\prime}} G^{\prime} \stackrel{f \times f^{\prime}}{\longrightarrow} H \\
& \text { and } \mathrm{L}\left(G \times f, f^{\prime} G^{\prime}\right)=\mathrm{L}(G) \cap \mathrm{L}\left(G^{\prime}\right) \text { for } H \text { unambiguous, } \\
& G \stackrel{f}{\longrightarrow} H \wedge G^{\prime} \stackrel{f^{\prime}}{\longrightarrow} H \Longrightarrow G \times f, f^{\prime} G^{\prime} \stackrel{f \times f^{\prime}}{\longrightarrow} H .
\end{aligned}
$$

Proof. 
Let $G \stackrel{f}{\longrightarrow} H$ and $G^{\prime} \stackrel{f^{\prime}}{\longrightarrow} H$. We denote $K=G \times{ }_{f, f^{\prime}} G^{\prime}$.

i) We have $K \subseteq G \times G^{\prime}$ so $K \stackrel{\mathrm{Id}}{\longrightarrow} G \times G^{\prime} \stackrel{\pi_{1}}{\longrightarrow} G \stackrel{f}{\longrightarrow} H$.

Hence $K \stackrel{\pi_{1} \circ f}{\longrightarrow} H$ i.e. $K \stackrel{f \times f^{\prime}}{\longrightarrow} H$.

Furthermore $\mathrm{L}(K) \subseteq \mathrm{L}\left(G \times G^{\prime}\right)=\mathrm{L}(G) \cap \mathrm{L}\left(G^{\prime}\right)$.

Let us prove the inverse inclusion using the hypothesis that $H$ is unambiguous.

Let $a_{1} \ldots a_{n} \in \mathrm{L}(G) \cap \mathrm{L}\left(G^{\prime}\right)$ with $n \geq 0$ and $a_{1}, \ldots, a_{n} \in T$.

There exists accepting paths $s_{0} \underset{{ }_{G}}{\stackrel{a_{1}}{\longrightarrow}} s_{1} \ldots \underset{G}{\stackrel{a_{n}}{\longrightarrow}} s_{n}$ and $s_{0}^{\prime} \underset{{ }_{G^{\prime}}}{\stackrel{a_{1}}{\longrightarrow}} s_{1}^{\prime} \ldots \frac{a_{n}}{{ }_{G^{\prime}}} s_{n}^{\prime}$ with $\iota s_{0}, o s_{n} \in G$ and $\iota s_{0}^{\prime}, o s_{n}^{\prime} \in G^{\prime}$.

So $f\left(s_{0}\right) \underset{H}{\stackrel{a_{1}}{\longrightarrow}} f\left(s_{1}\right) \ldots \underset{H}{\stackrel{a_{n}}{\longrightarrow}} f\left(s_{n}\right)$ and $f^{\prime}\left(s_{0}^{\prime}\right) \underset{H}{\stackrel{a_{1}}{\longrightarrow}} f^{\prime}\left(s_{1}^{\prime}\right) \ldots \underset{H}{\stackrel{a_{n}}{\longrightarrow}} f^{\prime}\left(s_{n}^{\prime}\right)$

with $\iota f\left(s_{0}\right), \iota f^{\prime}\left(s_{0}^{\prime}\right), o f\left(s_{n}\right), o f^{\prime}\left(s_{n}^{\prime}\right) \in H$.

As $H$ is unambiguous, $f\left(s_{0}\right)=f^{\prime}\left(s_{0}^{\prime}\right), \ldots, f\left(s_{n}\right)=f^{\prime}\left(s_{n}^{\prime}\right)$. Furthermore

$\left(s_{0}, s_{0}^{\prime}\right) \underset{G \times G^{\prime}}{\stackrel{a_{1}}{\longrightarrow}}\left(s_{1}, s_{1}^{\prime}\right) \ldots \underset{G \times G^{\prime}}{\stackrel{a_{n}}{\longrightarrow}}\left(s_{n}, s_{n}^{\prime}\right)$ with $\iota\left(s_{0}, s_{0}^{\prime}\right), o\left(s_{n}, s_{n}^{\prime}\right) \in G \times G^{\prime}$.

Thus $\left(s_{0}, s_{0}^{\prime}\right) \underset{K}{\stackrel{a_{1}}{\longrightarrow}}\left(s_{1}, s_{1}^{\prime}\right) \ldots \underset{K}{\stackrel{a_{n}}{\longrightarrow}}\left(s_{n}, s_{n}^{\prime}\right)$ with $\iota\left(s_{0}, s_{0}^{\prime}\right), o\left(s_{n}, s_{n}^{\prime}\right) \in K$.

Finally $a_{1} \ldots a_{n} \in \mathrm{L}(K)$.

ii) Let $G \stackrel{f}{\longrightarrow} H$ and $G^{\prime} \stackrel{f^{\prime}}{\longrightarrow} H$.

To show that $K \stackrel{f \times f^{\prime}}{\longrightarrow} H$, it remains to check that $K$ is $\left(f \times f^{\prime}\right)$-deterministic.

Let $\iota\left(s, s^{\prime}\right), \iota\left(t, t^{\prime}\right) \in K$ with $f(s)=f^{\prime}\left(s^{\prime}\right)=f(t)=f^{\prime}\left(t^{\prime}\right)$.

So $\iota s, \iota t \in G$ and $\iota s^{\prime}, \iota t^{\prime} \in G^{\prime}$.

As $G$ (resp. $\left.G^{\prime}\right)$ is $f\left(\right.$ resp $\left.f^{\prime}\right)$-deterministic, $s=t$ (resp. $\left.s^{\prime}=t^{\prime}\right)$.

Let $\left(r, r^{\prime}\right) \underset{K}{\stackrel{a}{\longrightarrow}}\left(s, s^{\prime}\right)$ and $\left(r, r^{\prime}\right) \underset{K}{\stackrel{a}{\longrightarrow}}\left(t, t^{\prime}\right)$ with $f(s)=f^{\prime}\left(s^{\prime}\right)=f(t)=f^{\prime}\left(t^{\prime}\right)$.

We have $r \underset{G}{\stackrel{a}{\longrightarrow}} s \wedge r \underset{G}{\stackrel{a}{\longrightarrow}} t$ and $r^{\prime} \underset{G^{\prime}}{\stackrel{a}{\longrightarrow}} s^{\prime} \wedge r^{\prime} \underset{G^{\prime}}{\stackrel{a}{\longrightarrow}} t^{\prime}$.

As $G$ (resp. $\left.G^{\prime}\right)$ is $f\left(\operatorname{resp} f^{\prime}\right)$-deterministic, $s=t\left(\right.$ resp. $\left.s^{\prime}=t^{\prime}\right)$. 


\section{B Stability of binary relation sets}

We introduce a condition of stability for any binary relation set $\mathcal{R}$ to get boolean algebras by structural recognizability for $\mathcal{F}(\mathcal{R})$ (Theorem B.1). Then we give a weaker stability condition to get boolean algebras by deterministic structural recognizability (Theorem B.2). Finally we show that structural recognizability coincide with deterministic structural recognizability for stable relation sets (Proposition B.3).

\section{B.a Strong stability}

We say that a binary relation set $\mathcal{R}$ is stable if

a) $\mathcal{R}$ is closed under $\cup, \cap,-,-1$, 。

b) for any $R \in \mathcal{R}$, the relation $\operatorname{Id}_{\operatorname{Dom}(R)}=\{(x, x) \mid x \in \operatorname{Dom}(R)\} \in \mathcal{R}$

c) for any functional relation $f \in \mathcal{R}$, there exists $R \in \mathcal{R}$ such that

- $\operatorname{Dom}(f) \cap \operatorname{Dom}(R)=\emptyset$,

- $R(x) \neq R\left(x^{\prime}\right)$ for any $x \neq x^{\prime} \in \operatorname{Dom}(R)$,

- $\{R(x) \mid x \in \operatorname{Dom}(R)\}=\left\{P \neq \emptyset \mid \exists z \in \operatorname{Im}(f), P \subseteq f^{-1}(z)\right\}$.

The last condition (c) called subset adding is illustrated by the following picture and says that for any element $z$ of $\operatorname{Im}(f)$ and any nonempty subset $P$ of $f^{-1}(z)$, there exists a unique $x$ of $\operatorname{Dom}(R)$ such that $R(x)=P$.

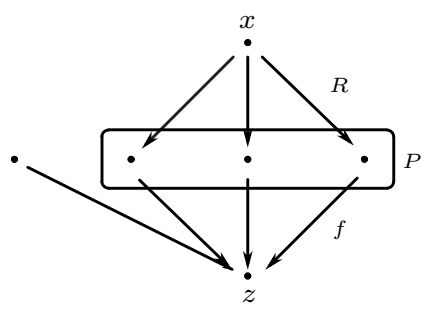

Let us give some explanations on this stability condition on $\mathcal{R}$. First this condition is on the vertices and edges of the automata in $\mathcal{F}(\mathcal{R})$ and not on the labels of the accepting paths. Thus the closure under - of condition (a) allows to remove vertices and edges and, of course, this does not correspond to the closure under difference of recognized languages. Furthermore the adding condition (c) allows to add vertices (memory) needed by determinization (exponential space for finite automata) and also by synchronization product (quadratic space for finite automata).

Under this condition of stability of $\mathcal{R}$, the automaton family $\operatorname{Aut}_{\mathcal{F}(\mathcal{R})}(H)$ is closed under morphic determinization, inversion and synchronization product.

Theorem B.1 For any stable family $\mathcal{R}$ of binary relations and for any unambiguous automaton $H \in \mathcal{F}(\mathcal{R})$, the language family $\operatorname{sRec}_{\mathcal{F}(\mathcal{R})}(H)$ is a boolean algebra with respect to $\mathrm{L}(H)$. 


\section{Proof.}

i) Let $G \in \mathcal{F}(\mathcal{R})$. Let us check that the identity

$$
\operatorname{Id}_{G}=\left\{(s, s) \mid s \in \mathrm{V}_{G}\right\}
$$

on the vertices of $G$ is in $\mathcal{R}$. As $\mathcal{R}$ is closed under $\cup$ and ${ }^{-1}$, the relation

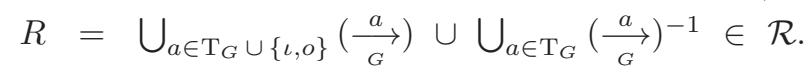

By the stability condition (b), $\mathcal{R}$ is closed under domain identity, hence

$$
\operatorname{Id}_{G}=\operatorname{Id}_{\operatorname{Dom}(R)} \in \mathcal{R} .
$$

ii) Let us show the following copy property:

for any $G \in \mathcal{F}(\mathcal{R})$ there exists $G^{\prime} \in \mathcal{F}(\mathcal{R})$

and an isomorphism in $\mathcal{R}$ between $G$ and $G^{\prime}$ such that $\mathrm{V}_{G} \cap \mathrm{V}_{G^{\prime}}=\emptyset$.

Let $G \in \mathcal{F}(\mathcal{R})$. By (i), $\operatorname{Id}_{G} \in \mathcal{R}$.

By the subset adding property (stability condition c) applied to $\operatorname{Id}_{G}$, there exists a relation $S \in \mathcal{R}$ such that $S(x) \neq S\left(x^{\prime}\right)$ for any $x \neq x^{\prime} \in \operatorname{Dom}(S)$ and

$$
\mathrm{V}_{G} \cap \operatorname{Dom}(S)=\emptyset \text { and }\{S(x) \mid x \in \operatorname{Dom}(S)\}=\left\{\{s\} \mid s \in \mathrm{V}_{G}\right\}
$$

So $S$ is a bijection from $\operatorname{Dom}(S)$ to $\mathrm{V}_{G}$.

We define the automaton $G^{\prime}$ for any $a \in \mathrm{T}_{G} \cup\{\iota, o\}$ by

$$
\underset{G^{\prime}}{\stackrel{a}{\longrightarrow}}=S \circ \underset{{ }_{G}}{\stackrel{a}{\longrightarrow}} \circ S^{-1} \in \mathcal{R} \text {. }
$$

Thus $G^{\prime} \in \mathcal{F}(\mathcal{R})$ and $G^{\prime}$ is isomorphic to $G$ by $S \in \mathcal{R}$.

iii) Let us show the closure of $\operatorname{sRec}_{\mathcal{F}(\mathcal{R})}(H)$ under intersection.

Let $G \stackrel{f}{\longrightarrow} H$ and $G^{\prime} \stackrel{f^{\prime}}{\longrightarrow} H$ with $G, G^{\prime} \in \mathcal{F}(\mathcal{R})$ and $f, f^{\prime} \in \mathcal{R}$.

As $H$ is unambiguous and by Lemma A.5,

$$
\mathrm{L}\left(G \times f, f^{\prime} G^{\prime}\right)=\mathrm{L}(G) \cap \mathrm{L}\left(G^{\prime}\right) .
$$

By the copy property (ii), we can assume that $\mathrm{V}_{G} \cap \mathrm{V}_{G^{\prime}}=\emptyset$.

By the subset adding property applied to $f \cup f^{\prime}$, there exists $S \in \mathcal{R}$ such that

$$
\begin{aligned}
& S(x) \neq S\left(x^{\prime}\right) \text { for any } x \neq x^{\prime} \in \operatorname{Dom}(S) \\
& \{S(x) \mid x \in \operatorname{Dom}(S)\}=\left\{P \neq \emptyset \mid \exists t \in \mathrm{V}_{H}\left(P \subseteq\left(f \cup f^{\prime}\right)^{-1}(t)\right\}\right.
\end{aligned}
$$

Let us restrict $\operatorname{Dom}(S)$ to its elements $x$ such that $S(x)$ has two vertices, one of $G$ (and the other of $G^{\prime}$ ): $\left|S(x) \cap \mathrm{V}_{G}\right|=\left|S(x) \cap \mathrm{V}_{G^{\prime}}\right|=1$.

This is realized by the following identity relation:

$$
I=\operatorname{Id}_{\operatorname{Dom}(S)}-\left[\left(S \circ\left(f \circ f^{-1}-\operatorname{Id}_{G}\right) \circ S^{-1}\right) \cup\left(S \circ\left(f^{\prime} \circ f^{\prime-1}-\operatorname{Id}_{G^{\prime}}\right) \circ S^{-1}\right)\right]
$$

So $I \in \mathcal{R}$ that we use to define the following restriction of $S$

$$
\widehat{S}=I \circ S \in \mathcal{R} \text {. }
$$

Let $\widehat{G}$ be the automaton defined for any $a \in \mathrm{T}_{H} \cup\{\iota, o\}$ by

$$
\underset{\widehat{G}}{\stackrel{a}{\longrightarrow}}=\left(\widehat{S} \circ \underset{G}{\stackrel{a}{\longrightarrow}} \circ \widehat{S}^{-1}\right) \cap\left(\widehat{S} \circ \underset{G^{\prime}}{\stackrel{a}{\longrightarrow}} \circ \widehat{S}^{-1}\right)
$$

Thus $\widehat{G} \in \mathcal{F}(\mathcal{R})$. This is illustrated by the following picture: 


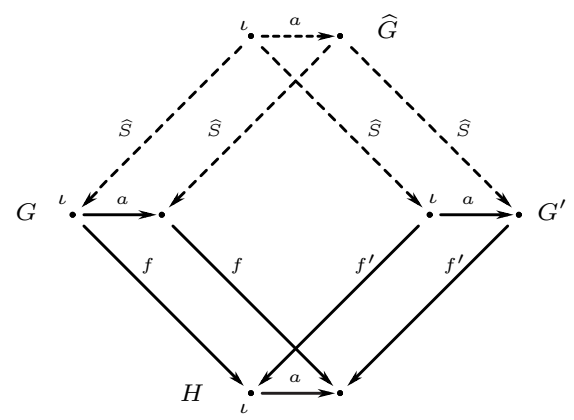

So $\widehat{G} \stackrel{\widehat{S} \circ f}{\longrightarrow} H$ with $\widehat{G}$ isomorphic to $G \times f, f^{\prime} G^{\prime}$.

Thus $\mathrm{L}(G) \cap \mathrm{L}\left(G^{\prime}\right)=\mathrm{L}(\widehat{G}) \in \operatorname{sRec}_{\mathcal{F}(\mathcal{R})}(H)$.

iv) Let $G \stackrel{f}{\longrightarrow} H$ with $G \in \mathcal{F}(\mathcal{R})$ and $f \in \mathcal{R}$.

Let us show that there exists $G^{\prime} \in \mathcal{F}(\mathcal{R})$ and $f^{\prime} \in \mathcal{R}$ such that $G^{\prime} \stackrel{f^{\prime}}{\longrightarrow} H$ and $G^{\prime}$ isomorphic to $\operatorname{Det}(G, f)$.

By the stability condition (c) applied to $f$, there exists $S \in \mathcal{R}$ such that

$$
\begin{aligned}
& S(x) \neq S\left(x^{\prime}\right) \text { for any } x \neq x^{\prime} \in \operatorname{Dom}(S) \\
& \{S(x) \mid x \in \operatorname{Dom}(S)\}=\Pi .
\end{aligned}
$$

Let us construct an automaton $G^{\prime}$ isomorphic to $\operatorname{Det}(G, f)$ such that

$$
\mathrm{V}_{G^{\prime}} \subseteq \operatorname{Dom}(S) \text { and } G^{\prime} \stackrel{S \circ f}{\longrightarrow} H .
$$

Let

$$
S_{0}=S \circ\left(\operatorname{Id}_{G}-\underset{G}{\stackrel{\iota}{\longrightarrow}}\right) \text { and } S_{1}=\left(S \circ f \circ f^{-1} \circ \underset{G}{\iota}\right)-S
$$

as illustrated below.

$(s)$

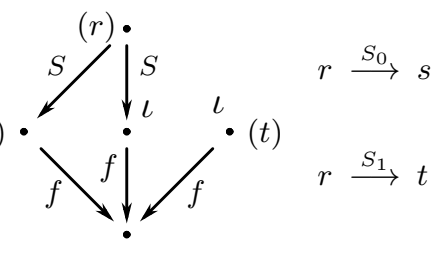

Thus $S_{0}, S_{1} \in \mathcal{R}$ hence

$$
\widehat{S}=S \circ f-\left(S_{0} \cup S_{1}\right) \circ f \in \mathcal{R}
$$

in such a way that $\operatorname{Dom}(\widehat{S})$ is the set of initial vertices of $G^{\prime}$ :

$$
\underset{{ }_{G^{\prime}}}{\stackrel{\iota}{\longrightarrow}}=\operatorname{Id}_{\operatorname{Dom}(\widehat{S})} \in \mathcal{R} \text {. }
$$

The $o$-transition of $G^{\prime}$ is defined by

$$
\underset{G^{\prime}}{\stackrel{o}{a}}=\operatorname{Id}_{\operatorname{Dom}(S)} \cap\left(S \circ \underset{G}{\stackrel{o}{\longrightarrow}} \circ S^{-1}\right) \in \mathcal{R} .
$$

Finally for any $a \in \mathrm{T}_{G}$, we define

$$
\begin{aligned}
\underset{G^{\prime}}{a} & =S \circ \stackrel{a}{\underset{G}{\longrightarrow}} \circ S^{-1} \\
& -S \circ \stackrel{a}{\longrightarrow} \circ\left(S \circ f \circ f^{-1}-S\right)^{-1} \\
& -\left[\left(S \circ \underset{G}{\stackrel{a}{\longrightarrow}} \circ f \circ f^{-1}\right)-(S \circ \underset{G}{\stackrel{a}{\longrightarrow}})\right] \circ S^{-1}
\end{aligned}
$$


which is illustrated below.

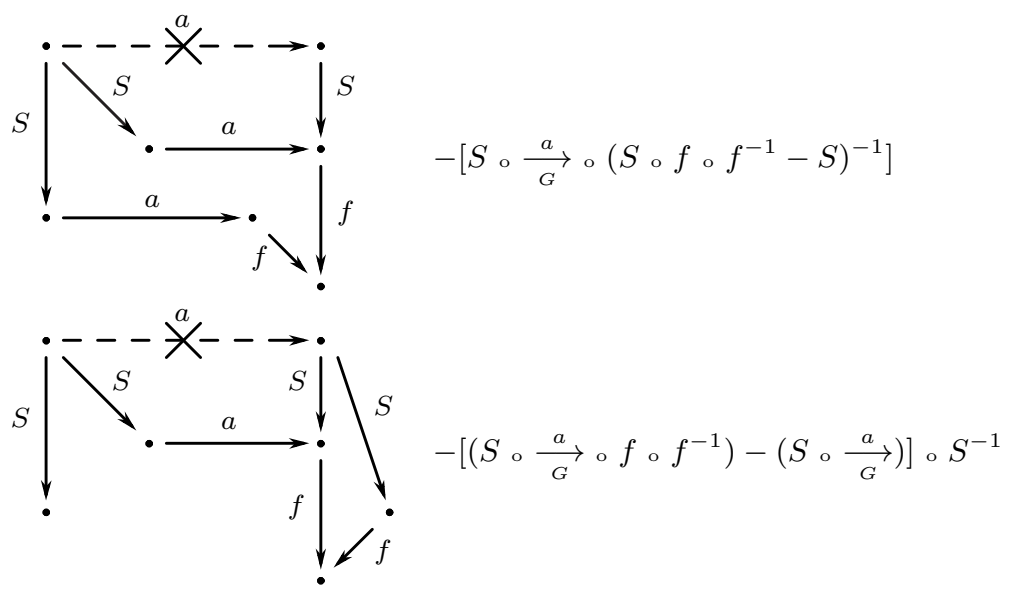

v) Let us show the closure of $\operatorname{sRec}_{\mathcal{F}(\mathcal{R})}(H)$ under complementation.

Let $G \stackrel{f}{\longrightarrow} H$ with $G \in \mathcal{F}(\mathcal{R})$ and $f \in \mathcal{R}$.

By the copy property (ii), we can assume that $\mathrm{V}_{G} \cap \mathrm{V}_{H}=\emptyset$.

By (iv), we can assume that $G$ is isomorphic to $\operatorname{Det}(G, f)$.

As $H$ is unambiguous and by Lemmas A.2 and A.4,

$$
\mathrm{L}(\operatorname{Inv}(G, f, H))=\mathrm{L}(H)-\mathrm{L}(G) .
$$

Let us show that there exists $g \in \mathcal{R}$ and $K \in \mathcal{F}(\mathcal{R})$ isomorphic to $\operatorname{Inv}(G, f, H)$ such that $K \stackrel{g}{\longrightarrow} H$.

We define such an automaton $K$ of vertex set $\mathrm{V}_{G} \cup \mathrm{V}_{H}$ as follows:

$$
\begin{aligned}
& \underset{K}{\stackrel{a}{\longrightarrow}}=\underset{G}{\stackrel{a}{\longrightarrow}} \cup \underset{H}{\stackrel{a}{\longrightarrow}} \cup\left(\left(f \circ \frac{a}{\longrightarrow}\right)-(\underset{G}{\longrightarrow} \circ f)\right) \quad \text { for any } a \in \mathrm{T}_{H} \\
& \underset{K}{\longrightarrow}=\underset{G}{\longrightarrow} \cup(\underset{H}{\longrightarrow}-f-1 \circ \underset{G}{\longrightarrow} \circ f) \\
& \underset{K}{\longrightarrow}=\underset{H}{\longrightarrow} \cup\left(\left(\operatorname{Id}_{G}-\underset{G}{\longrightarrow}\right) \cap\left(f \circ \underset{H}{\stackrel{o}{\longrightarrow}} \circ f^{-1}\right)\right)
\end{aligned}
$$

The construction of $K$ from $G \stackrel{f}{\longrightarrow} H$ is illustrated by the following picture:

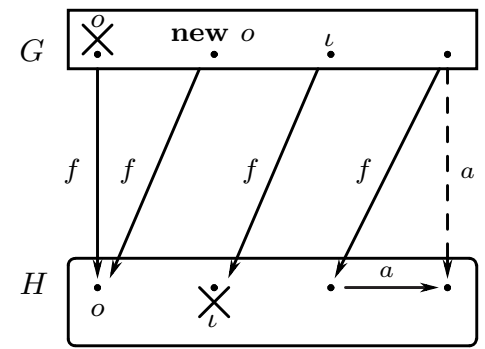

So $K \in \mathcal{F}(\mathcal{R})$ with $K$ isomorphic to $\operatorname{Inv}(G, f, H)$.

Furthermore $K \stackrel{g}{\longrightarrow} H$ for $g=f \cup \operatorname{Id}_{H} \in \mathcal{R}$.

Finally $\mathrm{L}(H)-\mathrm{L}(G)=\mathrm{L}(\operatorname{Inv}(G, f, H))=\mathrm{L}(K) \in \mathcal{F}(\mathcal{R})$. 


\section{B.b Weak stability}

To get boolean algebras by deterministic structural recognizability, we do not need the determinization operation. In fact the closure of $\operatorname{dsRec}_{\mathcal{F}(\mathcal{R})}(H)$ under complementation and intersection are obtained by closure of the automaton family

$$
\operatorname{dAut}_{\mathcal{F}(\mathcal{R})}(H)=\{G \in \mathcal{F}(\mathcal{R}) \mid \exists f \in \mathcal{R}(G \stackrel{f}{\longrightarrow} H)\}
$$

under inversion and synchronization product. We only need a weaker condition than stability on $\mathcal{R}$.

We say that $\mathcal{R}$ is weakly stable if in the stability condition, we replace the subset adding property (condition c) by the following pair adding property:

for any functional relation $f \in \mathcal{R}$, there exists $R \in \mathcal{R}$ such that

- $\operatorname{Dom}(f) \cap \operatorname{Dom}(R)=\emptyset$

- $R(x) \neq R\left(x^{\prime}\right)$ for any $x \neq x^{\prime} \in \operatorname{Dom}(R)$

- $\{R(x) \mid x \in \operatorname{Dom}(R)\}=\{\{y, f(y)\} \mid y \in \operatorname{Dom}(\mathrm{f})\}$.

The pair adding condition allows to add vertices needed for the synchronization product (quadratic space for finite automata) and also for the inversion (linear space for finite automata). Under this condition of weak stability of $\mathcal{R}$, the automaton family $\operatorname{dAut}_{\mathcal{F}(\mathcal{R})}(H)$ is closed under (morphic) inversion and synchronization product.

Theorem B.2 For any weakly stable family $\mathcal{R}$ of binary relations and for any unambiguous automaton $H \in \mathcal{F}(\mathcal{R})$, the language family $\operatorname{dsRec}_{\mathcal{F}(\mathcal{R})}(H)$ is a boolean algebra with respect to $\mathrm{L}(H)$.

\section{Proof.}

This is a simplification of the proof of Theorem B.1.

The part (i) remains valid for a weakly stable family, and also (ii) since the pair adding property applied to $\operatorname{Id}_{G}$ is sufficient.

The closure under intersection of (iii) remains true by Lemma A.5 and by applying directly the pair adding property to $f \circ f^{\prime-1}$.

The part (iv) is useless. The closure under complementation of (v) remains true by the second implication of Lemma A.4.

\section{B.c Strong and weak stability}

The deterministic structural recognizability coincides with structural recognizability for any stable relation family. 
Proposition B.3 For any stable family $\mathcal{R}$ and any automaton $H \in \mathcal{F}(\mathcal{R})$,

$$
\operatorname{dsRec}_{\mathcal{F}(\mathcal{R})}(H)=\operatorname{sRec}_{\mathcal{F}(\mathcal{R})}(H) .
$$

\section{Proof.}

By definition $\operatorname{dsRec}_{\mathcal{F}(\mathcal{R})}(H) \subseteq \operatorname{sRec}_{\mathcal{F}(\mathcal{R})}(H)$. Let us show the inverse inclusion. Let $L \in \operatorname{sRec}_{\mathcal{F}(\mathcal{R})}(H)$.

So $L=\mathrm{L}(G)$ for some $G \stackrel{f}{\longrightarrow} H$ with $G \in \mathcal{F}(\mathcal{R})$ and $f \in \mathcal{R}$.

By (iv) of the proof of Theorem B.1, there exists $G^{\prime} \in \mathcal{F}(\mathcal{R})$ and $f^{\prime} \in \mathcal{R}$ such that

$$
G^{\prime} \stackrel{f^{\prime}}{\longrightarrow} H \text { and } G^{\prime} \text { is isomorphic to } \operatorname{Det}(G, f) .
$$

By Lemma A.2, $G^{\prime} \stackrel{f^{\prime}}{\longrightarrow} H$ with $\mathrm{L}\left(G^{\prime}\right)=\mathrm{L}(\operatorname{Det}(G, f))=\mathrm{L}(G)$.

Hence $L=\mathrm{L}\left(G^{\prime}\right) \in \operatorname{dsRec}_{\mathcal{F}(\mathcal{R})}(H)$.

\section{Proof of Theorem 2}

By Theorem B.1, we get Theorem 2 by proving the stability of the families $\operatorname{Stack}(m, n)$ and $\operatorname{Count}(m, n)$ for each $m, n>0$.

The stability of the families $\operatorname{Stack}(m, n)$ are already known for $m=1$ except for the subset adding property. First, we show that for all these families, the subset adding property coincides with the pair adding which is easy to check. Then for each $n \geq 1$, we translate by desynchronization product the closure properties of $\operatorname{Stack}(1, n)$ to $\operatorname{Stack}(m, n)$ for any $m>1$.

\section{C.a From pair adding to subset adding}

Having a linear order $<_{N}$ on a set $N$ of symbols, recall that the length lexicographic order $<_{\text {llex }}$ on the set $N^{*}$ of words over $N$ is the linear order defined for any $a, b \in N$ and $u, v, w \in N^{*}$ by

$$
u<_{\text {llex }} v \text { for }|u|<|v| \text {; uav }<_{\text {llex }} u b w \text { for } a<_{N} b \text { and }|v|=|w| \text {. }
$$

This order is extended to the set $\left(N^{*}\right)^{+}$of non empty word tuples as follows: for any $m, n>0$ and $u_{1}, \ldots, u_{m}, v_{1}, \ldots, v_{n} \in N^{*}$,

$$
\left(u_{1}, \ldots, u_{m}\right)<_{\text {llex }}\left(v_{1}, \ldots, v_{n}\right) \text { for } u_{1} \# \ldots \# u_{m}<\text { llex } v_{1} \# \ldots \# v_{n}
$$

where the symbol $\# \notin N$ and $\#<a$ for any $a \in N$.

Weak stability of a family $\mathcal{R}$ of binary relations on $\left(N^{*}\right)^{+}$coincides with stability when the relations of $\mathcal{R}$ are bounded and $\mathcal{R}$ is closed under intersection with $<_{\text {llex }}$.

Proposition C.1 Let $\mathcal{R}$ be a weakly stable family of bounded relations on $\left(N^{*}\right)^{+}$which is closed under intersection with $<_{\text {lex }}$. Then $\mathcal{R}$ is stable. 


\section{Proof.}

We have to show that $\mathcal{R}$ satisfies the subset adding property.

i) Let us extend the pair adding property to any not functional relation of $\mathcal{R}$.

Let $R \in \mathcal{R}$. Let us show that there exists $S \in \mathcal{R}$ such that

$\operatorname{Dom}(R) \cap \operatorname{Dom}(S)=\emptyset$ and $S(x) \neq S\left(x^{\prime}\right)$ for any $x \neq x^{\prime} \in \operatorname{Dom}(S)$

and $\{S(x) \mid x \in \operatorname{Dom}(S)\}=\{\{y, z\} \mid y R z\}$.

As $R$ is bounded, we take the integer

$$
b=\max \{|R(x)| \mid x \in \operatorname{Dom}(R)\} .
$$

Let us check that for any $1 \leq i \leq b$, the function

$$
f_{i}=\left\{(x, y) \in R||\left\{z \leq_{\text {lex }} y \mid z \in R(x)\right\} \mid=i\right\} \in \mathcal{R} .
$$

This is done by strong induction on $1 \leq i \leq b$.

Basic case: $i=1$. So

$$
f_{1}=R-\left(R \circ<_{\text {llex }}\right)=R-R \circ\left[\left(R^{-1} \circ R\right) \cap<_{\text {llex }}\right] \in \mathcal{R} .
$$

Inductive case: $\{1, \ldots, i-1\} \Longrightarrow(i>1)$.

So $f_{i}$ is the function of the basic case for $R-\left(f_{1} \cup \ldots \cup f_{i-1}\right)$.

It remains to take

$$
S=S_{1} \cup \ldots \cup S_{b}
$$

for $S_{1}, \ldots, S_{b}$ defined by induction as follows:

$i=1$ : we take $S_{1}$ by pair adding from $f_{1}$.

$i>1$ : having taken $S_{1}, \ldots, S_{i-1}$ with $\operatorname{Dom}(S), \operatorname{Dom}\left(S_{1}\right), \ldots, \operatorname{Dom}\left(S_{i-1}\right)$ pairwise disjoint, we take $S_{i}^{\prime}$ by pair adding from $f_{i} \cup \operatorname{Id}_{\operatorname{Dom}\left(S_{1} \cup \ldots \cup S_{i-1}\right)}$, and we define

$$
S_{i}=S_{i}^{\prime}-\left(S_{i}^{\prime} \circ \operatorname{Id}_{\operatorname{Dom}\left(S_{1} \cup \ldots \cup S_{i-1}\right)}\right)
$$

ii) Let $R \in \mathcal{R}$ and $b=\max \left\{\left|R^{-1}(z)\right| \mid z \in \operatorname{Im}(R)\right\}$.

Let us show the subset adding property for $R$ : there exists $S \in \mathcal{R}$ such that

$\operatorname{Dom}(R) \cap \operatorname{Dom}(S)=\emptyset$ and $S(x) \neq S\left(x^{\prime}\right)$ for any $x \neq x^{\prime} \in \operatorname{Dom}(S)$

and $\{S(x) \mid x \in \operatorname{Dom}(S)\}=\left\{P \neq \emptyset \mid \exists z \in \operatorname{Im}(R), P \subseteq R^{-1}(z)\right\}$.

Let $\bar{R}=\left(R \circ R^{-1}\right) \cap<$ llex .

The single adding is realized by $S_{1} \in \mathcal{R}$ taken by extended pair adding (i) from $\operatorname{Id}_{\operatorname{Dom}(R)}$.

Let $R_{1}=\emptyset$. By induction on $1<i \leq b$, we define

$$
R_{i}=\left[S_{i-1}-\left(S_{i-1} \circ R_{i-1}^{-1}\right)\right] \circ \bar{R}
$$

and like in (i), we take $S_{i}^{\prime}$ by extended pair adding (i) from $R_{i} \cup \operatorname{Id}_{\operatorname{Dom}\left(S_{1} \cup \ldots \cup S_{i-1}\right)}$, and we define

$$
S_{i}=S_{i}^{\prime}-\left(S_{i}^{\prime} \text { 。 } \operatorname{Id}_{\operatorname{Dom}\left(S_{1} \cup \ldots \cup S_{i-1}\right)}\right) .
$$

Finally we take $S=S_{1} \cup \ldots \cup S_{b}$.

Proposition C.1 is used to show the subset adding property for any family $\mathcal{S}$ tack $(m, n)$ by only checking the pair adding property. 


\section{C.b Stability}

Let us show the stability of each family $\mathcal{S} \operatorname{tack}(m, n)$. The stability of $\mathcal{C}$ ount $(m, n)$ is similar. We begin with $m=n=1$.

Proposition C.2 The family Stack is stable.

\section{Proof.}

The proof of stability condition (a) has already been done in [3]. However we give here a complete proof.

i) We say that two words $u, v$ are left-irreducible if

$$
u=\varepsilon \vee v=\varepsilon \vee(u, v \neq \varepsilon \wedge u(1) \neq v(1))
$$

meaning that they cannot have a first common letter. By taking the greatest common prefix $z$ of any words $u, v$, we have the unique decomposition

$$
u=z x \wedge v=z y \text { for } x, y \text { left-irreducible. }
$$

In that case and for any language $W$,

$$
W(u, v)=W z(x, y)
$$

meaning that any couple $(w u, w v)$ for $w \in W$ is obtained by applying by suffix the rule $(x, y)$ under the left context $w z$. This is illustrated by the unlabelled arrow in the following $\operatorname{Alph}(W \cup\{u, v\})$-tree:

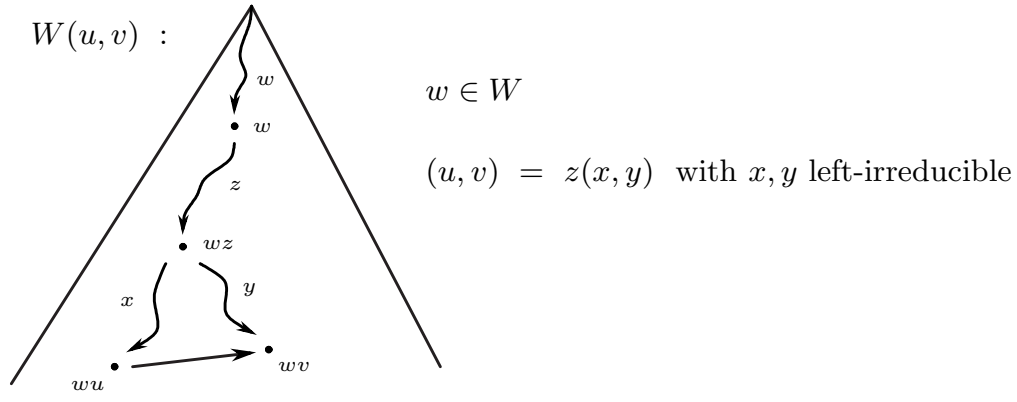

We say that a system $R$ is left-irreducible if $u, v$ are left-irreducible for any $(u, v) \in R$. For any left-irreducible systems $R, S$ and for any languages $W, Z$, let us show the following equalities:

$$
\begin{aligned}
W . R \cap Z . S & =(W \cap Z) \cdot(R \cap S) \\
W . R-Z . S & =(W-Z) \cdot R \cup(W \cap Z)(R-S) \\
W . R \circ Z . S & =\bigcup\left\{\left(W w^{-1} \cap Z\right)(w u, y) \mid(u, v) \in R \wedge(w v, y) \in S\right\} \\
& \cup \bigcup\left\{\left(W \cap Z z^{-1}\right)(u, z y) \mid(u, z x) \in R \wedge(x, y) \in S\right\}
\end{aligned}
$$

ii) Let us check Equality (1) for $R$ and $S$ left-irreducible.

$\supseteq$ : obvious and for any relations $R, S$.

$\subseteq$ : let $w(u, v)=z(x, y)$ with $w \in W, z \in Z,(u, v) \in R$ and $(x, y) \in S$.

So $w u=z x$ and $w v=z y$.

We distinguish the three complementary cases below.

Case 1: $|w|<|z|$. Thus $u(1)=z(|w|+1)=v(1)$. 
Such a case is not possible since $u, v$ are left-irreducible.

Case 2: $|w|>|z|$. Thus $x(1)=w(|z|+1)=y(1)$.

Such a case is not possible since $x, y$ are left-irreducible.

Case 3: $|w|=|z|$. Thus $w=z \in W \cap Z$ and $(u, v)=(x, y) \in R \cap S$.

iii) Let us check Equality (2) for $R$ and $S$ left-irreducible. We have

$$
\begin{aligned}
W . R & =[(W \cap Z) \cup(W-Z)] \cdot R \\
& =(W \cap Z) \cdot R \cup(W-Z) \cdot R \\
& =(W \cap Z) \cdot[(R \cap S) \cup(R-S)] \cup(W-Z) \cdot R \\
& =(W \cap Z) \cdot(R \cap S) \cup(W \cap Z) \cdot(R-S) \cup(W-Z) \cdot R
\end{aligned}
$$

which is by (i) a partition of W.R.

Furthermore by (i), $Z . S$ is disjoint of $(W \cap Z) .(R-S) \cup(W-Z) . R$.

As $(W \cap Z) .(R \cap S) \subseteq Z$.S, we get Equality (2).

iv) Let us check Equality (3) for any relations $R, S$.

$\subseteq$ : Let $w \in W, z \in Z,(u, v) \in R$ and $(x, y) \in S$ such that $w v=z x$.

We have to check that ( $w u, z y$ ) belongs to the right hand side of Equality (3).

We distinguish the two cases below.

Case 1: $|w| \geq|z|$.

By Levi's lemma, there exists $\bar{w}$ such that $w=z \bar{w}$ and $x=\bar{w} v$.

So $(w u, z y)=z(\bar{w} u, y)$ with $z \in W \bar{w}^{-1} \cap Z,(u, v) \in R$ and $(\bar{w} v, y) \in S$.

Case 2: $|w| \leq|z|$.

By Levi's lemma, there exists $\bar{z}$ such that $z=w \bar{z}$ and $v=\bar{z} x$.

So $(w u, z y)=w(u, \bar{z} y)$ with $w \in W \cap Z \bar{z}^{-1},(u, \bar{z} x) \in R$ and $(x, y) \in S$.

$\supseteq$ : Due to the union of the set of the right hand side of Equality (3), we have the two cases below.

Case 1: Let $z \in W w^{-1} \cap Z,(u, v) \in R$ and $(w v, y) \in S$.

As $z w \in W$, we get $(z w u, z w v) \in W . R$.

As $z \in Z$, we get $(z w v, z y) \in Z . S$ hence $(z w u, z y) \in W . R \circ Z . S$.

Case 2: Let $w \in W \cap Z z^{-1},(u, z x) \in R$ and $(x, y) \in S$.

As $w \in W$, we get $(w u, w z x) \in W . R$.

As $w z \in Z$, we get $(w z x, w z y) \in Z . S$ hence $(w u, w z y) \in W . R \circ Z . S$.

v) We can show the stability of the binary relation family $\mathcal{S}$ tack.

By definition, the family $\mathcal{S}$ tack is closed under $\cup$.

Equalities (1-3) give the closure under $\cap,-,{ }^{-1}$, o for the family of elementary suffix relations. In fact for $\{(u, v),(x, y)\}$ left-irreducible and for languages $W, Z$, we get 


$$
\begin{aligned}
& W(u, v) \cap Z(x, y)= \begin{cases}(W \cap Z)(u, v) & \text { if }(u, v)=(x, y) \\
\emptyset & \text { otherwise }\end{cases} \\
& W(u, v)-Z(x, y)= \begin{cases}(W-Z)(u, v) & \text { if }(u, v)=(x, y) \\
W(u, v) & \text { otherwise }\end{cases} \\
& W(u, v) \circ Z(x, y)= \begin{cases}\left(W w^{-1} \cap Z\right)(w u, y) & \text { if } x=w v \\
\left(W \cap Z z^{-1}\right)(u, z y) & \text { if } v=z x \\
\emptyset & \text { otherwise. }\end{cases}
\end{aligned}
$$

Furthermore

$$
(W(u, v))^{-1}=W(v, u) .
$$

It follows the closure under $\cap,-,{ }^{-1}$, o of $\mathcal{S}$ tack.

In fact consider any suffix relations $R, S$ :

$$
R=\bigcup_{i=1}^{p} W_{i}\left(u_{i}, v_{i}\right) \text { and } S=\bigcup_{j=1}^{q} Z_{j}\left(x_{j}, y_{j}\right)
$$

for some $p, q \geq 0$ with for any $1 \leq i \leq p, W_{i}$ is regular and $u_{i}, v_{i}$ are leftirreducible, and for any $1 \leq j \leq q, Z_{j}$ is regular and $x_{j}, y_{j}$ are left-irreducible. Thus

$$
\begin{aligned}
R \cap S & =\bigcup_{i, j} W_{i}\left(u_{i}, v_{i}\right) \cap Z_{j}\left(x_{j}, y_{j}\right) \quad ; R \circ S=\bigcup_{i, j} W_{i}\left(u_{i}, v_{i}\right) \circ Z_{j}\left(x_{j}, y_{j}\right) \\
R-S & =\bigcup_{i} \bigcap_{j} W_{i}\left(u_{i}, v_{i}\right)-Z_{j}\left(x_{j}, y_{j}\right) ; \quad R^{-1}=\bigcup_{i}\left(W_{i}\left(u_{i}, v_{i}\right)\right)^{-1} \\
\operatorname{Id}_{\operatorname{Dom}(R)} & =\bigcup_{i=1}^{p} W_{i}\left(u_{i}, u_{i}\right)
\end{aligned}
$$

It remains to check the closure of $\mathcal{S}$ tack under subset adding. Note that

$$
R \cap<_{\text {llex }}=\bigcup\left\{W_{i}\left(u_{i}, v_{i}\right) \mid 1 \leq i \leq p \wedge u_{i}<_{\text {llex }} v_{i}\right\} .
$$

By Proposition C.1, it remains to check the weak stability property.

As the family of elementary suffix relations is closed under $\cap$ and -, we can assume that $\left(W_{i}\left(u_{i}, v_{i}\right)\right)_{1 \leq i \leq p}$ is a partition of $R$ i.e.

$$
W_{i}\left(u_{i}, v_{i}\right) \cap W_{j}\left(u_{j}, v_{j}\right)=\emptyset \text { for any } 1 \leq i<j \leq p .
$$

We take $p$ new symbols $\&_{1}, \ldots, \&_{p}$ and a relation $S$ satisfying the pair adding property for $R$ is the following:

$$
S=\bigcup_{i=1}^{p} W_{i}\left(\&_{i}, u_{i}\right) \cup \bigcup_{i=1}^{p} W_{i}\left(\&_{i}, v_{i}\right)
$$

which is a relation of $\mathcal{S}$ tack.

Let us translate the stability of $\mathcal{S} \operatorname{tack}(1,1)$ to $\mathcal{S} \operatorname{tack}(m, 1)$ for every $m>1$.

Proposition C.3 The family $\operatorname{Stack}(m, 1)$ is stable for each $m>1$.

\section{Proof.}

We restrict the proof to $m=2$ since the generalization to any $m>2$ is simple. Recall that a relation of $\mathcal{S} \operatorname{tack}(2,1)$ is a finite union of elementary relations of the form

$$
\begin{aligned}
& \left(W_{1}, W_{2}\right)\left(\left(u_{1}, u_{2}\right) \longrightarrow\left(v_{1}, v_{2}\right)\right) \\
= & \left\{\left(w_{1} u_{1}, w_{2} u_{2}\right) \longrightarrow\left(w_{1} v_{1}, w_{2} v_{2}\right) \mid w_{1} \in W_{1} \wedge w_{2} \in W_{2}\right\}
\end{aligned}
$$


with $W_{1}, W_{2} \in \operatorname{Reg}\left(N^{*}\right)$ and $u_{1}, u_{2}, v_{1}, v_{2} \in N^{*}$.

The translation of the stability of $\mathcal{S} \operatorname{tack}(1,1)$ to $\mathcal{S} \operatorname{tack}(2,1)$ is done by the following operation:

$$
(u, v) \otimes(x, y)=((u, x),(v, y)) \text { for any } u, v, x, y \in N^{*}
$$

that we extend by union to any binary relations $R, S$ on $N^{*}$ to get the following binary relation $R \otimes S$ on $N^{*} \times N^{*}$ :

$$
R \otimes S=\{((u, x),(v, y)) \mid u R v \wedge x S y\} .
$$

We have the following equalities:

$$
\begin{aligned}
\left(W_{1}, W_{2}\right)\left(\left(u_{1}, u_{2}\right) \longrightarrow\left(v_{1}, v_{2}\right)\right) & =W_{1}\left(u_{1} \longrightarrow v_{1}\right) \otimes W_{2}\left(u_{2} \longrightarrow v_{2}\right) \\
(R \otimes S) \cap\left(R^{\prime} \otimes S^{\prime}\right) & =\left(R \cap R^{\prime}\right) \otimes\left(S \cap S^{\prime}\right) \\
(R \otimes S)-\left(R^{\prime} \otimes S^{\prime}\right) & =\left(R-R^{\prime}\right) \otimes S \cup R \otimes\left(S-S^{\prime}\right) \\
(R \otimes S) \circ\left(R^{\prime} \otimes S^{\prime}\right) & =\left(R \circ R^{\prime}\right) \otimes\left(S \circ S^{\prime}\right)
\end{aligned}
$$

Let us check the first equality.

$$
\begin{aligned}
& W_{1}\left(u_{1} \longrightarrow v_{1}\right) \otimes W_{2}\left(u_{2} \longrightarrow v_{2}\right) \\
= & \left\{\left(w_{1} u_{1}, w_{1} v_{1}\right) \mid w_{1} \in W_{1}\right\} \otimes\left\{\left(w_{2} u_{2}, w_{2} v_{2}\right) \mid w_{2} \in W_{2}\right\} \\
= & \left\{\left(w_{1} u_{1}, w_{2} u_{2}\right) \longrightarrow\left(w_{1} v_{1}, w_{2} v_{2}\right) \mid w_{1} \in W_{1} \wedge w_{2} \in W_{2}\right\} \\
= & \left(W_{1}, W_{2}\right)\left(\left(u_{1}, u_{2}\right) \longrightarrow\left(v_{1}, v_{2}\right)\right) .
\end{aligned}
$$

Let us check the second equality.

$$
\begin{aligned}
& (R \otimes S) \cap\left(R^{\prime} \otimes S^{\prime}\right) \\
= & \{((u, x),(v, y)) \mid u R v \wedge x S y\} \cap\left\{((u, x),(v, y)) \mid u R^{\prime} v \wedge x S^{\prime} y\right\} \\
= & \left\{((u, x),(v, y)) \mid(u, v) \in R \cap R^{\prime} \wedge(x, y) \in S \cap S^{\prime}\right\} \\
= & \left(R \cap R^{\prime}\right) \otimes\left(S \cap S^{\prime}\right) .
\end{aligned}
$$

Let us check the third equality.

$$
\begin{aligned}
& (R \otimes S)-\left(R^{\prime} \otimes S^{\prime}\right) \\
= & \{((u, x),(v, y)) \mid u R v \wedge x S y\}-\left\{((u, x),(v, y)) \mid u R^{\prime} v \wedge x S^{\prime} y\right\} \\
= & \left\{((u, x),(v, y)) \mid u R-R^{\prime} v \wedge x S y\right\} \\
\cup & \left\{((u, x),(v, y)) \mid u R v \wedge x S-S^{\prime} y\right\} \\
= & \left(R-R^{\prime}\right) \otimes S \cup R \otimes\left(S-S^{\prime}\right) .
\end{aligned}
$$

Let us check the last equality.

$$
\begin{aligned}
& (R \otimes S) \circ\left(R^{\prime} \otimes S^{\prime}\right) \\
= & \{((u, x),(v, y)) \mid u R v \wedge x S y\} \circ\left\{((v, y),(w, z)) \mid v R^{\prime} w \wedge y S^{\prime} z\right\} \\
= & \left\{((u, x),(w, z)) \mid u R \circ R^{\prime} w \wedge x S \circ S^{\prime} z\right\} \\
= & \left(R \circ R^{\prime}\right) \otimes\left(S \circ S^{\prime}\right) .
\end{aligned}
$$

These four equalities permit to translate the closure under $\cap,-$, o of $\mathcal{S} \operatorname{tack}(1,1)$ to $\mathcal{S} \operatorname{tack}(2,1)$.

For the other stability closure properties, consider any relation $R$ of $\mathcal{S}$ tack $(2,1)$ :

$$
R=\bigcup_{i=1}^{p}\left(W_{i}, W_{i}^{\prime}\right)\left(\left(u_{i}, u_{i}^{\prime}\right) \longrightarrow\left(v_{i}, v_{i}^{\prime}\right)\right)
$$

with for any $1 \leq i \leq p, W_{i}, W_{i}^{\prime} \in \operatorname{Reg}\left(N^{*}\right)$ and $u_{i}, u_{i}^{\prime}, v_{i}, v_{i}^{\prime} \in N^{*}$. So

$$
R^{-1}=\bigcup_{i=1}^{p}\left(W_{i}^{\prime}, W_{i}\right)\left(\left(u_{i}^{\prime}, u_{i}\right) \longrightarrow\left(v_{i}^{\prime}, v_{i}\right)\right)
$$


and

$$
\operatorname{Id}_{\operatorname{Dom}(R)}=\bigcup_{i=1}^{p}\left(W_{i}, W_{i}^{\prime}\right)\left(\left(u_{i}, u_{i}^{\prime}\right) \longrightarrow\left(u_{i}, u_{i}^{\prime}\right)\right)
$$

As

$$
\begin{aligned}
& R \cap<_{\text {llex }} \\
= & \bigcup\left\{\left(W_{i}^{\prime}, W_{i}\right)\left(\left(u_{i}^{\prime}, u_{i}\right) \longrightarrow\left(v_{i}^{\prime}, v_{i}\right)\right) \mid 1 \leq i \leq p \wedge\left(u_{i}, u_{i}^{\prime}\right)<_{\text {llex }}\left(v_{i}, v_{i}^{\prime}\right)\right\}
\end{aligned}
$$

and by Proposition C.1, it remains to check the closure under pair adding.

By the closure under $\cap$ and -, we can assume that

$$
\left(\left(W_{i}^{\prime}, W_{i}\right)\left(\left(u_{i}^{\prime}, u_{i}\right) \longrightarrow\left(v_{i}^{\prime}, v_{i}\right)\right)\right)_{1 \leq i \leq p} \text { is a partition of } R \text {. }
$$

We take $p$ new symbols $\&_{1}, \ldots, \&_{p}$ and a relation $S$ satisfying the pair adding property for $R$ is the following:

$$
S=\bigcup_{i=1}^{p}\left(W_{i}, W_{i}^{\prime}\right)\left(\left(\&_{i}, \&_{i}\right) \longrightarrow\left(u_{i}, u_{i}^{\prime}\right)\right) \cup \bigcup_{i=1}^{p}\left(W_{i}, W_{i}^{\prime}\right)\left(\left(\&_{i}, \&_{i}\right) \longrightarrow\left(v_{i}, v_{i}^{\prime}\right)\right)
$$

which is a relation of $\mathcal{S} \operatorname{tack}(2,1)$.

For any $n \geq 2$, the closure of $\mathcal{S} \operatorname{tack}(1, n)$ under $\cap,-$, 。 (and the iteration of 。) has been done in [2] and its extension to $\mathcal{S} \operatorname{tack}(m, n)$ for any $m>1$ is given by the equalities in the proof of Proposition C.3. By Proposition C.1, the subset adding property follows from the pair adding property.

\section{Proof of Theorems 3 and 4}

By Theorem B.2, we get Theorems 3 and 4 by proving the weak stability of the family $\mathcal{S} y n c$ of synchronized (rational) relations and its subfamily bSync of bounded difference length synchronized relations.

Proposition D.1 The families Sync and bSync are weakly stable.

Proof.

Recall that a synchronized relation is a finite union of elementary synchronized relations of the form:

$$
R(U, V) \text { with } R \in \operatorname{Reg}\left((N \times N)^{*}\right) \text { and } U, V \in \operatorname{Reg}\left(N^{*}\right) .
$$

Such an elementary relation is in normal form if

$$
(U=V=\{\varepsilon\}) \text { or }(U=\{\varepsilon\} \wedge \varepsilon \notin V) \text { or }(V=\{\varepsilon\} \wedge \varepsilon \notin U) .
$$

i) Let us check that any synchronized relation is a finite union of elementary synchronized relations in normal form.

It is sufficient to check it for $U \times V$ with $U, V \in \operatorname{Reg}\left(N^{*}\right)$.

The relation accepted by an automaton $C$ labelled in $N \times N$ is

$$
\mathrm{R}(C)=\left\{\left(a_{1} \ldots a_{n}, b_{1} \ldots b_{n}\right) \mid n \geq 0 \wedge\left(a_{1}, b_{1}\right) \ldots\left(a_{n}, b_{n}\right) \in \mathrm{L}(C)\right\} .
$$

Given finite automata $A$ and $B$ recognizing respectively $U$ and $V$, we define the finite automaton

$$
C=\{(p, s) \stackrel{(a, b)}{\longrightarrow}(q, t) \mid p \underset{A}{\stackrel{a}{\longrightarrow}} q \wedge s \underset{B}{\stackrel{b}{\longrightarrow}} t\} \cup\{\iota(p, s) \mid \iota p \in A \wedge \iota s \in B\}
$$


For any $p \in \mathrm{V}_{A}$ and $s \in \mathrm{V}_{b}$, we define the automata

$$
\begin{aligned}
A_{p} & =\left(A-\{\iota\} \times \mathrm{V}_{A}\right) \cup\{\iota p\} \text { and } B_{s}=\left(B-\{\iota\} \times \mathrm{V}_{B}\right) \cup\{\iota s\} \\
C_{p, s} & =C \cup\{o(p, s)\} .
\end{aligned}
$$

This allows to describe the following decomposition of $U \times V$ :

$$
\begin{aligned}
U \times V & =\bigcup_{o p \in A, o s \in B} \mathrm{R}\left(C_{p, s}\right)(\{\varepsilon\},\{\varepsilon\}) \\
& \cup \bigcup_{o p \in A, s \in \mathrm{V}_{B}} \mathrm{R}\left(C_{p, s}\right)\left(\{\varepsilon\}, \mathrm{L}\left(B_{s}\right)-\{\varepsilon\}\right) \\
& \cup \bigcup_{p \in \mathrm{V}_{A}, o s \in B} \mathrm{R}\left(C_{p, s}\right)\left(\mathrm{L}\left(A_{p}\right)-\{\varepsilon\},\{\varepsilon\}\right)
\end{aligned}
$$

as a finite union of elementary synchronized relations in normal form.

ii) Let us check the closure of $\mathcal{S} y n c$ under $\cap$ and - .

By definition $\mathcal{S} y n c$ is closed under $U$ and by distributivity, it remains to check the closure under $\cap$ and - for elementary synchronized relations in normal form. In that case,

$$
\begin{aligned}
R(U, \varepsilon) \cap S(V, \varepsilon) & =(R \cap S)(U \cap V, \varepsilon) \\
R(U, \varepsilon) \cap S(\varepsilon, V) & =\emptyset=R(U, \varepsilon) \cap S \\
R(U, \varepsilon)-S(V, \varepsilon) & =(R-S)(U, \varepsilon) \cup R(U-V, \varepsilon) \\
R(U, \varepsilon)-S(\varepsilon, V) & =R(U, \varepsilon)=R(U, \varepsilon)-S
\end{aligned}
$$

iii) Let us check the closure of $\mathcal{S} y n c$ under o. We have

$$
R(U, V) \circ S(X, Y)=\left(R\left(\varepsilon, V X^{-1}\right) \circ S\right)(U, Y) \cup\left(R \circ S\left(X V^{-1}, \varepsilon\right)\right)(U, Y)
$$

Furthermore

$$
R(\varepsilon, U) \circ S=\bigcup_{u \in U} \bigcup_{z \in N|u|}\left(R \circ S(u, z)^{-1}\right)(\varepsilon, z)
$$

which can be described by a finite union since the length-preserving synchronized relation $S$ has a finite number of (right) residuals, and

$$
\left\{\left(u^{\prime}, z^{\prime}\right) \mid S(u, z)^{-1}=S\left(u^{\prime}, z^{\prime}\right)^{-1}\right\}
$$

is a length-preserving synchronized relation.

iv) Let us check the weak stability conditions (b) and (c) for Sync.

The closure under inverse is obvious:

$$
(R(U, V))^{-1}=R^{-1}(V, U)
$$

and also the closure under domain identity:

$$
\operatorname{Id}_{\operatorname{Dom}(R(U, V))}=\operatorname{Id}_{\operatorname{Dom}(R) \cdot U} .
$$

v) It remains to show the pair adding property for any $R \in \mathcal{S}$ ync.

Let $N_{R}$ be the subset of symbols of $N$ used in $R$.

We take an injective mapping

$$
\varphi:\left(N_{R} \cup\{\varepsilon\}\right) \times\left(N_{R} \cup\{\varepsilon\}\right) \hookrightarrow N-N_{R}
$$

that we extend to an injective mapping $N_{R}^{*} \times N_{R}^{*} \longrightarrow\left(N-N_{R}\right)^{*}$ by induction as follows: for any $u, v \in N_{R}^{*}$ and $a, b \in N_{R}$,

$$
\begin{aligned}
\varphi(a u, b v) & =\varphi(a, b) \varphi(u, v) \\
\varphi(a u, \varepsilon) & =\varphi(a, \varepsilon) \varphi(u, \varepsilon) \\
\varphi(\varepsilon, b v) & =\varphi(\varepsilon, b) \varphi(\varepsilon, v) \\
\varphi(\varepsilon, \varepsilon) & =\varepsilon
\end{aligned}
$$


It remains to check that

$$
S=\{(\varphi(u, v), u) \mid u R v\} \cup\{(\varphi(u, v), v) \mid u R v\}
$$

is a relation in $\mathcal{S} y n c$.

The relation $R$ is recognized by a synchronized transducer $A$ i.e. a finite automaton labelled in $\left(N_{R} \times N_{R}\right) \cup\left(N_{R} \times\{\varepsilon\}\right) \cup\left(\{\varepsilon\} \times N_{R}\right)$ such that for any $p \underset{A}{\stackrel{(a, b)}{\longrightarrow}} q \underset{A}{\stackrel{(c, d)}{\longrightarrow}} r$, if $a=\varepsilon($ resp. $b=\varepsilon)$ then $c=\varepsilon$ (resp. $d=\varepsilon$ ).

Let $A_{1}$ (resp. $A_{2}$ ) be the automaton obtained from $A$ by renaming each label $(a, b)$ by $(\varphi(a, b), a)$ [resp. $(\varphi(a, b), b)]$. Then

$$
S=\mathrm{R}\left(A_{1}\right) \cup \mathrm{R}\left(A_{2}\right) \in \text { Sync. }
$$

vi) Note that the bounded length difference property is preserved by $\cup, \llbracket \mathbb{},-$, , , ${ }^{-1}$. Furthermore in (v) and for $R$ of difference length bounded by $b$, the relation $S$ is also of difference length bounded by $b$ since $|\varphi(u, v)|=\max \{|u|,|v|\}$.

In consequence the relation subfamily bSync remains weakly stable.

\section{E Proof of Propositions 5.1}

This proposition is based on the length recognizability. Recall that for any family $\mathcal{R}$ of binary relations on words, the length recognizability from an automaton $H$ with word vertices, is the following language family:

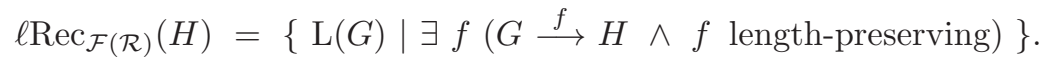

The length structural recognizability from $H$ according to $\mathcal{R}$ is

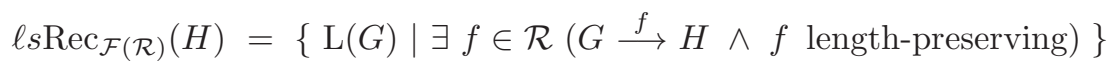

the recognizability by morphisms in $\mathcal{R}$ preserving the length.

For the family bSync of binary relations on words, structural recognizability can be done by length preserving morphisms.

Proposition E.1 For any automaton $H$ with $\mathrm{V}_{H} \subset N^{+}$,

$$
\operatorname{sRec}_{\mathcal{F}(\mathrm{b} \mathcal{S} y n c)}(H)=\ell_{\operatorname{sRec}}{\operatorname{F}\left(\mathrm{b} \mathcal{S}_{\text {ync }}\right)}(H) \text {. }
$$

\section{Proof.}

$\supseteq$ : immediate.

$\subseteq$ : Let $L \in \operatorname{sRec}_{\mathcal{F}\left(\text { b } \mathcal{S}_{\text {ync }}\right)}(H)$.

There is a reduction $G \stackrel{f}{\longrightarrow} H$ such that $G \in \mathcal{F}$ (bSync), $f \in$ bS $\mathcal{S}$ ync, $L=\mathrm{L}(G)$. As $f \in$ bSync which is closed under $\cup, \cap,-$ we can express $f$ by a disjoint union of elementary synchronized relations of bounded length difference:

$$
f=\biguplus_{i=1}^{n} f_{i}\left(u_{i} \longrightarrow v_{i}\right)
$$

where for any $1 \leq i \leq n, f_{i} \in \operatorname{Reg}\left((N \times N)^{*}\right)$ functional, $u_{i} \in N^{*}$ and $v_{i} \in N^{+}$. We take $n$ new symbols $p_{1}, \ldots, p_{n}$ and we define the relation 


$$
g=\bigcup_{i=1}^{n} \operatorname{Id}_{\operatorname{Dom}\left(f_{i}\right)}\left(\left.u_{i} \longrightarrow\right|^{\left|v_{i}\right|-1} p_{i}\right) .
$$

So $g \in$ bSync and $\operatorname{Dom}(g)=\operatorname{Dom}(f)=\mathrm{V}_{G}$.

Let us check that this relation $g$ is functional and injective. Let

$$
\left(x_{i} u_{i},\left.x_{i}\right|^{\left|v_{i}\right|-1} p_{i}\right),\left(x_{j}^{\prime} u_{j},\left.x_{j}^{\prime}\right|^{\left|v_{j}\right|-1} p_{j}\right) \in g
$$
that

with $1 \leq i, j \leq n, x_{i} \in \operatorname{Dom}\left(f_{i}\right)$ and $x_{j}^{\prime} \in \operatorname{Dom}\left(f_{j}\right)$. We have to prove

Assume that $x_{i} u_{i}=x_{j}^{\prime} u_{j}$. So

$$
x_{i} u_{i}=\left.x_{j}^{\prime} u_{j} \Longleftrightarrow x_{i}\right|^{\left|v_{i}\right|-1} p_{i}=\left.x_{j}^{\prime}\right|^{\left|v_{j}\right|-1} p_{j} .
$$

$$
\left(x_{i} u_{i}, f\left(x_{i} u_{i}\right)\right)=\left(x_{j}^{\prime} u_{j}, f\left(x_{j}^{\prime} u_{j}\right)\right) \in f_{i} .\left(u_{i}, v_{i}\right) \cap f_{j} \cdot\left(u_{j}, v_{j}\right) .
$$

Thus $i=j$ hence $u_{i}=u_{j}$ and $x_{i}=x_{j}^{\prime}$. So $\left.x_{i}\right|^{\left|v_{i}\right|-1} p_{i}=\left.x_{j}^{\prime}\right|^{\left|v_{j}\right|-1} p_{j}$.

Assume that $\left.x_{i}\right|^{\left|v_{i}\right|-1} p_{i}=\left.x_{j}^{\prime}\right|^{\left|v_{j}\right|-1} p_{j}$.

So $p_{i}=p_{j}$ hence $i=j$. Thus $v_{i}=v_{j}$ and $x_{i}=x_{j}^{\prime}$. So $x_{i} u_{i}=x_{j}^{\prime} u_{j}$.

So $G$ is isomorphic to the automaton

$$
g(G)=\{g(s) \stackrel{a}{\longrightarrow} g(t) \mid s \underset{G}{\stackrel{a}{\longrightarrow}} t\} \cup\{c g(s) \mid c s \in G\} .
$$

Furthermore $g(G) \in \mathcal{F}$ (bSync) since for any $a \in \mathrm{T}_{g(G)} \cup\{\iota, o\}=\mathrm{T}_{G} \cup\{\iota, o\}$,

$$
\underset{g(G)}{\stackrel{a}{\longrightarrow}}=g^{-1} \circ \underset{G}{\stackrel{a}{\longrightarrow}} \circ g \text {. }
$$

For $f^{\prime}=g^{-1} \circ f$, we have

$$
g(G) \stackrel{f^{\prime}}{\longrightarrow} H \text { and } f^{\prime} \in \text { bSync is length-preserving. }
$$

Thus $L=\mathrm{L}(G)=\mathrm{L}(g(G)) \in \ell \operatorname{sRec}_{\mathcal{F}\left(\text { bS } \mathcal{S}_{\text {ync }}\right)}(H)$.

We characterize the family $\operatorname{Idl}_{\mathcal{S} \text { ync }}(\vec{T})$ of input-driven synchronized languages by structural recognizibility from the input-driven automaton $\operatorname{Inp}_{1,1}(\vec{T})$ according to the family $\mathcal{F}$ (bS ync) of bounded length difference synchronized automata.

Proposition 5.1 For any partition $\vec{T}$,

$$
\operatorname{sRec}_{\mathcal{F}(b \text { Sync })}\left(\operatorname{Inp}_{1,1}(\vec{T})\right)=\operatorname{Idl}_{\mathcal{S}_{\text {ync }}(\vec{T}) .}
$$

\section{Proof.}

For Inp $_{1,1}$, the partition has three parts: $\vec{T}=\left(\mathrm{T}_{-1}, \mathrm{~T}_{0}, \mathrm{~T}_{1}\right)$.

$\supseteq$ : Let $L \in \operatorname{Idl}_{\mathcal{S}_{\text {ync }}}(\vec{T})$.

So $L=\mathrm{L}(G)$ for some automaton $G$ with $\mathrm{V}_{G} \subset N^{+}$such that

$$
\underset{G}{\stackrel{a}{\rightarrow}} \in \mathcal{S} \text { ync for any } a \in \mathrm{T}_{G} \cup\{\iota, o\}
$$

and for any $u \underset{G}{\stackrel{a}{\longrightarrow}} v$, we have $|u|,|v|>0$ with

$$
\left(|v|=|u|+i \text { for } a \in T_{i}\right) \text { and }(|u|=|v|=1 \text { for } a=\iota) .
$$


In particular $G \in \mathcal{F}$ (b $\mathcal{S}$ ync).

Furthermore the length preserving mapping $f:\left.\mathrm{V}_{G} \longrightarrow\right|^{*} \kappa$ i.e.

$$
f(u)=\left.\right|^{|u|-1} \kappa \text { for any } u \in \mathrm{V}_{G}
$$

is a morphism from $G$ into $\operatorname{Inp}_{1,1}(\vec{T}): G \stackrel{f}{\longrightarrow} \operatorname{Inp}_{1,1}(\vec{T})$.

As $\mathrm{V}_{G}$ is a regular language, $f \in$ bSync.

Thus $L=\mathrm{L}(G) \in \operatorname{sRec}_{\mathcal{F}(\mathrm{b} \mathcal{S} \text { ync) }}\left(\operatorname{Inp}_{1,1}(\vec{T})\right)$.

ii) Let $L \in \operatorname{sRec}_{\mathcal{F}\left(\text { b } \mathcal{S}_{\text {ync }}\right)}\left(\operatorname{Inp}_{1,1}(\vec{T})\right)$.

By Lemma E.1, $L \in \ell \operatorname{sRec}_{\mathcal{F}(\text { b } \mathcal{S y n c})}\left(\operatorname{Inp}_{1,1}(\vec{T})\right)$.

So $L=\mathrm{L}(G)$ for some $G \stackrel{f}{\longrightarrow} \operatorname{Inp}_{1,1}(\vec{T})$ such that

$G \in \mathcal{F}$ (bSync) and $f$ length-preserving.

So $G$ is input-driven for bSync hence $L \in \operatorname{Idl}_{\mathcal{S}_{\text {ync }}}(\vec{T})$.

\section{F $\quad$ Proof of Proposition 3.1}

We consider the language

$$
\begin{aligned}
L=\left\{a^{n} c b^{n_{1}} a^{n_{1}} d \ldots c b^{n_{k}} a^{n_{k}} d \mid n, k>0\right. & \wedge 0<n_{1}, \ldots, n_{k} \leq n \\
& \left.\wedge \exists 1 \leq i<j \leq k, n_{i}=n_{j}\right\} .
\end{aligned}
$$

It is easy to check that

$$
L \in \ell \operatorname{Rec}_{\mathcal{F}\left(\mathcal{S} \operatorname{tack}_{2}\right)}\left(\operatorname{Inp}_{1,2}(\{b\}, \emptyset,\{a\},\{d\},\{c\})\right) .
$$

We take the alphabet

$$
N=\{\mid, \dagger\} \cup Q
$$

where $\mid, \dagger$ are the two stack letters and $Q$ is a finite set of states.

Taking states $p, q, r, s, t, f \in Q$, we define a stack of stack automaton for having the following derivations:

$$
\left.p \stackrel{a^{m+n+1}}{\longrightarrow}||^{m} \dagger\right|^{n} q \stackrel{c}{\longrightarrow}\left(\left.\left.\right|^{m} \dagger\right|^{n},\left.\left.\right|^{m} \dagger\right|^{n}\right) r
$$

with

and

$$
\left(\left.\left.\right|^{m} \dagger\right|^{n},\left.\left.\right|^{m} \dagger\right|^{n}\right) r \stackrel{b^{i} a^{i} d c}{\longrightarrow}\left(\left.\left.\right|^{m} \dagger\right|^{n},\left.\left.\right|^{m} \dagger\right|^{n}\right) r \quad \text { for any } \quad 0 \leq i \leq m+n+1
$$

$$
\left(\left.||^{m} \dagger\right|^{n},\left.\left.\right|^{m} \dagger\right|^{n}\right) r \stackrel{b^{n+1} a^{n+1} d c}{\longrightarrow}\left(\left.\left.\right|^{m} \dagger\right|^{n},\left.\left.\right|^{m} \dagger\right|^{n}\right) s
$$

From state $s$, these two last derivations are repeated by

$$
\left(\left.\left.\right|^{m} \dagger\right|^{n},\left.\left.\right|^{m} \dagger\right|^{n}\right) s \stackrel{b^{i} a^{i} d c}{\longrightarrow}\left(\left.\left.\right|^{m} \dagger\right|^{n},\left.\left.\right|^{m} \dagger\right|^{n}\right) s \quad \text { for any } 0 \leq i \leq m+n+1
$$

$$
\left.\left.\left(\left.\left.\right|^{m} \dagger\right|^{n},\left.\left.\right|^{m} \dagger\right|^{n}\right) s \stackrel{b^{n+1} a^{n+1} d}{\longrightarrow}\right|^{m} \dagger\right|^{n} f \stackrel{c}{\longrightarrow}\left(\left.\left.\right|^{m} \dagger\right|^{n},\left.\left.\right|^{m} \dagger\right|^{n}\right) t
$$


Finally it remains to realize the following derivations:

$$
\left.\left(\left.||^{m} \dagger\right|^{n},\left.\left.\right|^{m} \dagger\right|^{n}\right) t \stackrel{b^{i} a^{i} d}{\longrightarrow}\right|^{m} \dagger^{n} f \quad \text { for any } 0 \leq i \leq m+n+1 .
$$

The unique initial configuration is $p$ and the set of final configurations is $\left.\right|^{*} \dagger^{*} f$. It remains to show that the complement $\bar{L}$ of $L$ satisfies

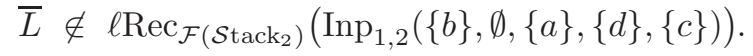

Assume the converse hence the language

$$
\begin{aligned}
M=\left\{a^{n} c b^{n_{1}} a^{n_{1}} d \ldots c b^{n_{k}} a^{n_{k}} d \mid n, k>0\right. & \wedge 0<n_{1}, \ldots, n_{k} \leq n \\
& \left.\wedge \forall 1 \leq i<j \leq k, n_{i} \neq n_{j}\right\}
\end{aligned}
$$

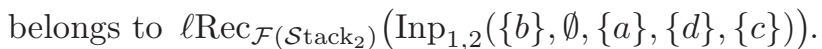

So there is a stack of stack automaton $G$ recognizing $\mathrm{L}(G)=M$ and lengthreducible to $\operatorname{Inp}_{1,2}(\{b\}, \emptyset,\{a\},\{d\},\{c\})$.

Let $N$ be the alphabet of the configurations of $G$ and

$$
O p=N \cup \bar{N} \cup\{\#, \overline{\#}, !, ?\} .
$$

For any $a \in T \cup\{\iota, o\}$, we have

$$
\underset{G}{\stackrel{a}{\longrightarrow}}=\bigcup_{i \in I_{a}} \llbracket W_{i}^{a}\left(u_{i}^{a}, v_{i}^{a}\right) \rrbracket
$$

for some finite set $I_{a}$ and for each $i \in I_{a}, W_{i}^{a} \in \operatorname{Reg}\left(O p^{*}\right)$ and $u_{i}^{a}, v_{i}^{a} \in O p^{*}$. So

$$
\vec{x} \underset{G}{\stackrel{a}{\longrightarrow}} \vec{y} \Longrightarrow \vec{x} \underset{G}{\stackrel{\overline{u_{i}^{a}} v_{i}^{a}}{\longrightarrow}} \vec{y} \quad \text { for some } i \in I_{a}
$$

Let

$$
\beta=\max \left\{\left|u_{i}^{a}\right|+\left|v_{i}^{a}\right| \mid a \in T \cup\{\iota, o\} \wedge i \in I_{a}\right\}
$$

be the maximal length of the rules defining the labelled transitions of $G$.

We decompose any word $u \in N^{*}$ into

$$
u=\operatorname{Pre}(u) \cdot \operatorname{Suf}(u) \quad \text { with }|\operatorname{Suf}(u)|=\min (|u|, \beta) .
$$

Note that

$$
\vec{x} \stackrel{\#(N \cup \bar{N})^{*}}{\longrightarrow} \vec{y} \quad \Longrightarrow \quad \vec{x}=\vec{y}
$$

and

$$
\left(x_{0}, \ldots, x_{k}\right) \stackrel{\#(N \cup \bar{N})^{i}}{\longrightarrow} \vec{y} \quad \Longrightarrow \quad \vec{y}=\left(x_{0}, \ldots, x_{k-2}, y_{k-1}, y_{k}\right)
$$

with $x_{k-1}=x_{k}$ and $y_{k-1}=y_{k}$ of same prefix of length $\max \left(\left|x_{k}\right|,\left|y_{k}\right|\right)-i$.

In particular we get for any $u, v \in N^{*}$ and $n \geq 0$,

$$
u \stackrel{c b^{n} a^{n} d}{\longrightarrow} v \Longrightarrow(|u|=|v| \wedge \operatorname{Pre}(u)=\operatorname{Pre}(v)) .
$$

Let $\gamma=|N|^{\beta}$ and $a^{\gamma} c b^{n_{1}} a^{n_{1}} d \ldots c b^{n_{\gamma}} a^{n_{\gamma}} d \in M$.

There exists an accepting path of $G$ of the form

$$
u \stackrel{a^{\gamma}}{\longrightarrow} v_{0} \stackrel{c b^{n_{1}} a^{n_{1}} d}{\longrightarrow} v_{1} \ldots \stackrel{c b^{n_{\gamma} a^{n_{\gamma}}} \longrightarrow}{\longrightarrow} v_{\gamma} \quad \text { with } \iota u, o v_{\gamma} \in G .
$$


As $\left|v_{0}\right|=\ldots=\left|v_{\gamma}\right|$ and $\operatorname{Pre}\left(v_{0}\right)=\ldots=\operatorname{Pre}\left(v_{\gamma}\right)$, there exists $0<i<j \leq \gamma$ such that $v_{i}=v_{j}$. Thus

$$
a^{\gamma} c b^{n_{1}} a^{n_{1}} d \ldots c b^{n_{j}} a^{n_{j}} d c b^{n_{i+1}} a^{n_{i+1}} d \ldots c b^{n_{\gamma}} a^{n_{\gamma}} d \in M
$$

which brings a contradiction since the factor $c b^{n_{j}} a^{n_{j}} d$ appears twice. 